\title{
DNS of Flows over Periodic Hills using a Discontinuous-Galerkin Spectral-Element Method
}

\author{
Laslo T. Diosady*and Scott M. Murman ${ }^{\dagger}$ \\ NASA Ames Research Center, Moffett Field, CA, USA
}

\begin{abstract}
Direct numerical simulation (DNS) of turbulent compressible flows is performed using a higher-order space-time discontinuous-Galerkin finite-element method. The numerical scheme is validated by performing DNS of the evolution of the Taylor-Green vortex and turbulent flow in a channel. The higher-order method is shown to provide increased accuracy relative to low-order methods at a given number of degrees of freedom. The turbulent flow over a periodic array of hills in a channel is simulated at Reynolds number 10,595 using an 8th-order scheme in space and a 4 th-order scheme in time. These results are validated against previous large eddy simulation (LES) results. A preliminary analysis provides insight into how these detailed simulations can be used to improve Reynoldsaveraged Navier-Stokes (RANS) modeling.
\end{abstract}

\section{Introduction}

Prediction of separated flows about curved bodies remains a significant challenge in computational fluid dynamics (CFD). These flows involve large-scale unsteady motion of the separation and reattachment points, and are generally poorly predicted by current Reynolds averaged Navier-Stokes (RANS) models. The development of new RANS models for these types of flows will rely on validation with respect to detailed data obtained through direct numerical simulation (DNS) on representative test-cases, which capture the main flow features. In this work we present DNS results for the separated flow over periodic hills using a new unstructured higher-order scheme for compressible high-Reynolds number flows.

The flow over an array of hills in a channel has been used as a benchmark case for the study of separation. It is modeled using a domain which is periodic in the stream-wise and span-wise directions, simplifying the boundary conditions in a numerical simulation. Periodic hills were initially examined experimentally by Almeida et al. ${ }^{1}$ at Reynolds numbers of up to 60,000 based on the hill height. In this work we consider the geometry presented by Mellen et al. ${ }^{2}$ This geometry retains the complex flow features from the original experiments of Almeida et al. in a smaller domain for the numerical simulation, and the corresponding experimental data more closely match the periodic approximation.

The periodic hills geometry has been investigated both numerically and experimentally over a wide range of Reynolds numbers. ${ }^{2-5}$ Fröhlich et al. ${ }^{3}$ performed highly resolved incompressible large eddy simulation (LES) at Reynolds number, $R e=10,595$ using two different 2nd-order finite-volume discretizations with different subgrid models. Breuer et al. ${ }^{5}$ present a comprehensive review of large eddy simulation (LES) and DNS performed to date, comparing experimental data with numerical results using DNS up to $R e=5600$ and LES up to $R e=10,595$. The current work extends these studies to DNS at $R e=10,595$.

Higher-order methods have been shown to be more efficient for simulations requiring high spatial and temporal resolution, allowing for solutions with fewer degrees of freedom and lower computational cost than traditional second-order CFD methods. ${ }^{6}$ As this work is focused on subsonic flows without shocks, the exact solution is in $C^{\infty}$, and thus we do not expect the convergence rate of a higher-order scheme to be limited by solution irregularity. Spectral methods were employed for the first DNS calculations in periodic domains. ${ }^{7}$ However, as DNS has been performed for increasingly more complex geometries other numerical methods

\footnotetext{
*Postdoctoral Fellow, Oak Ridge Associated Universities, laslo.diosady@nasa.gov

†Scott.M.Murman@nasa.gov
} 
such as finite-difference and finite-volume methods have become more common. In this work, we use a spacetime discontinuous Galerkin (DG) finite-element method, which extends to an arbitrary order of accuracy. Finite-element methods are well suited to problems with complex geometry, where unstructured meshes may be required. Higher-order finite-element methods are particularly attractive due to the possibility of using local $h$ - and $p$-adaptation. The use of a space-time formulation allows for adaptation in both the spatial and temporal directions, similar to adaptive mesh refinement (AMR) methods with sub-cycling.

This paper begins with a brief description of the governing equations and our space-time discretization in Section II. In Sections III and IV we present initial validation of our numerical scheme by performing direct numerical simulation of the Taylor-Green vortex evolution and a fully developed turbulent channel flow. In Section $\mathrm{V}$ we present results from the direct numerical simulation of the periodic hill problem. Finally, we present a summary and discussion in Section VI.

\section{Numerical Method}

The compressible Navier-Stokes equations are written in conservative form as:

$$
\begin{aligned}
\rho_{, t}+\left(\rho u_{i}\right)_{, i} & =0 \\
\left(\rho u_{j}\right)_{, t}+\left(\rho u_{i} u_{j}+p \delta_{i j}\right)_{, i} & =\tau_{i j, i} \\
(\rho E)_{, t}+\left(\rho u_{i} H\right)_{, i} & =\left(\tau_{i j} v_{j}+\kappa_{T} T_{, j} \delta_{i j}\right)_{, i}
\end{aligned}
$$

where $\rho$ is the density, $u_{j}$ are the components of the velocity, $E$ is the total energy, $p$ is the static pressure, $H=E+\frac{p}{\rho}$ is the total enthalpy, $\tau_{i j}$ is the viscous stress tensor, $\kappa_{T}$ is the thermal conductivity, $T=p / \rho R$ is the temperature, and $R$ is the gas constant. The pressure is given by:

$$
p=(\gamma-1)\left(\rho E-\frac{1}{2} \rho v_{k} v_{k}\right)
$$

where $\gamma$ is the specific heat ratio. The viscous stress tensor, $\tau_{i j}$, is given by:

$$
\tau_{i j}=\mu\left(v_{i, j}+v_{j, i}\right)-\lambda v_{k, k} \delta_{i j}
$$

where $\mu$ is the viscosity, $\lambda=\frac{2}{3} \mu$ is the bulk viscosity and $\delta_{i j}$ the Kronecker delta. We rewrite (1)-(3) as a single vector equation:

$$
\boldsymbol{u}_{, t}+\left(\boldsymbol{F}_{i}^{I}-\boldsymbol{F}_{i}^{V}\right)_{, i}=0
$$

where $\boldsymbol{u}=\left[\rho, \rho u_{j}, \rho E\right]$ is the conservative state vector, while $\boldsymbol{F}_{i}^{I}$ and $\boldsymbol{F}_{i}^{V}$ are, respectively, the inviscid and viscous fluxes. Applying a change of variables $\boldsymbol{u}=\boldsymbol{u}(\mathbf{v})$, where $\mathbf{v}$ are the entropy variables, the Navier-Stokes equations may be rewritten as:

$$
A_{0} \mathbf{v}_{, t}+A_{i} \mathbf{v}_{, x_{i}}-\left(K_{i j} \mathbf{v}_{, x_{j}}\right)_{, x_{i}}=0
$$

with symmetric $A_{0}=\boldsymbol{u}_{, \mathbf{v}}, A^{i}=\boldsymbol{F}_{i, \boldsymbol{u}}^{I} A_{0}=\boldsymbol{F}_{i, \mathbf{v}}^{I}$ and $K_{i j}=\boldsymbol{F}_{i, \boldsymbol{u}_{, x_{j}}}^{V} A_{0}=\boldsymbol{F}_{i, \mathbf{v}, x_{j}}^{V} \cdot{ }^{8}$ The entropy variables are:

$$
\mathbf{v}=\left[\begin{array}{c}
-\frac{s}{\gamma-1}+\frac{\gamma+1}{\gamma-1}-\frac{\rho E}{p} \\
\frac{\rho u_{j}}{p} \\
-\frac{\rho}{p}
\end{array}\right]
$$

where $s=\log \left(p / \rho^{\gamma}\right)$ is the entropy.

We proceed to discretize (7) as follows. The domain, $\Omega$, is partitioned into non-overlapping hexahedral elements, $\kappa$, while the time is partitioned into time intervals (time-slabs), $I^{n}=\left[t^{n}, t^{n+1}\right]$. Define $\mathcal{V}_{h}=$ $\left\{\boldsymbol{w},\left.\boldsymbol{w}\right|_{\kappa} \in[\mathcal{P}(\kappa \times I)]^{5}\right\}$, the space-time finite-element space consisting of piece-wise polynomial functions in both space and time on each element. We seek a solution $\mathbf{v} \in \mathcal{V}_{h}$ which satisfies the weak form:

$\sum_{\kappa}\left\{\int_{I} \int_{\kappa}-\left(\boldsymbol{w}_{, t} \boldsymbol{u}+\boldsymbol{w}_{, x_{i}}\left(\boldsymbol{F}_{i}^{I}-\boldsymbol{F}_{i}^{V}\right)\right)+\int_{I} \int_{\partial \kappa} \boldsymbol{w}\left(\widehat{\boldsymbol{F}_{i}^{I} \boldsymbol{n}_{i}}-\widehat{\boldsymbol{F}_{i}^{V} \boldsymbol{n}_{i}}\right)+\int_{\kappa} \boldsymbol{w}\left(t_{-}^{n+1}\right) \boldsymbol{u}\left(t_{-}^{n+1}\right)-\boldsymbol{w}\left(t_{+}^{n}\right) \boldsymbol{u}\left(t_{-}^{n}\right)\right\}=0$ 
for all $\boldsymbol{w} \in \mathcal{V}_{h}$. Here $\widehat{\boldsymbol{F}_{i}^{I} \boldsymbol{n}_{i}}$ and $\widehat{\boldsymbol{F}_{i}^{V} \boldsymbol{n}_{i}}$ denote numerical flux functions approximating the inviscid and viscous fluxes, respectively. In this work, the inviscid flux is computed using the method of Ismail and Roe ${ }^{9}$, while the viscous flux is computed using the method of Bassi and Rebay. ${ }^{10}$

The space, $\mathcal{V}_{h}$, is spanned by the tensor product of $1 \mathrm{D}$ nodal Lagrange basis functions defined at the Gauss-Legendre points. Integrals appearing in (9) are approximated with numerical quadrature using a polynomial dealiasing rule with twice as many quadrature points as solution points in each coordinate direction. With the choice of basis and quadrature rule, (9) gives a system of nonlinear equations which must solved for each time-slab. In this work we use the preconditioned Jacobian-free Newton-Krylov solver previously presented. ${ }^{11}$

\section{Taylor-Green Vortex Problem}

Initial validation of our numerical method was performed using DNS of the evolution of the Taylor-Green vortex. The Taylor-Green vortex evolution is used as a model problem for turbulent flow as it involves only periodic boundary conditions, no forcing and a simple initial condition. The flow is solved on an isotropic domain, which spans $[0,2 \pi L]$ in each coordinate direction. The initial conditions are given by:

$$
\begin{aligned}
u & =V_{0} \sin (x / L) \cos (y / L) \cos (z / L) \\
v & =-V_{0} \cos (x / L) \sin (y / L) \cos (z / L) \\
w & =0 \\
p & \left.=\rho_{0} V_{0}^{2}\left[\frac{1}{\gamma M_{0}^{2}}+\frac{1}{16}(\cos (2 x)+\cos (2 y))(\cos (2 z)+2)\right)\right]
\end{aligned}
$$

where $u, v$ and $w$ are the components of the velocity in the $x$-, $y$ - and $z$-directions, $p$ is the pressure and $\rho$ is the density. The Taylor-Green vortex flow is simulated using the compressible Navier-Stokes equations at $M_{0}=0.1$ and $R e=\frac{\rho_{0} V_{0} L}{\mu}=1600$. The flow is initialized to be isothermal $\left(\frac{p}{\rho}=\frac{p_{0}}{\rho_{0}}=R T_{0}\right)$.

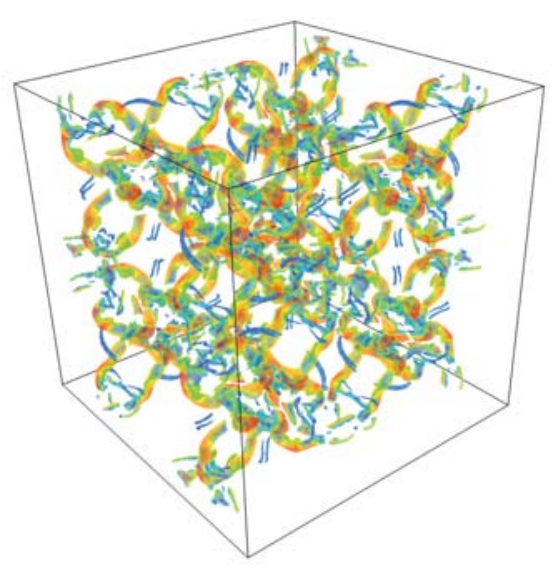

Figure 1. Iso-contours of vorticity magnitude at peak dissipation for the Taylor-Green vortex evolution at $M=0.1, R e=1600$, computed using $256^{3}$ degrees of freedom.

Starting from the simple initial condition, the flow becomes turbulent through repeated vortex stretching leading to progressively smaller eddies, which are then dissipated to heat through the action of molecular viscosity. Figure 1 shows the iso-contours of vorticity magnitude at the point of peak dissipation from a 16thorder solution. The figure highlights the presence of small vortical structures captured by the higher-order scheme.

For each simulation the temporal evolution of the kinetic energy

$$
E_{k}=\frac{1}{\Omega} \int_{\Omega} \frac{1}{2} \rho \mathbf{v} \cdot \mathbf{v} d \Omega
$$


is monitored. The evolution of the kinetic energy dissipation rate $\epsilon=-\frac{d E_{k}}{d t}$ was computed from the data at the space-time quadrature points. Figure 2 shows the temporal evolution of the kinetic energy dissipation rate computed with 256 degrees of freedom in each coordinate direction using 2nd- 4th-, 8th-, 16th-order spatial discretizations with a 4th-order temporal discretization. The corresponding meshes have, respectively, 128, 64, 32 and 16 elements in each coordinate direction. Reference data computed from an incompressible simulation using a spectral code on a $512^{3} \operatorname{grid}^{12}$ is also presented. For the 2nd-order scheme, there remains significant numerical dissipation at this resolution and the point of peak dissipation is poorly captured. With increasing polynomial order, the results relative to the spectral data are significantly improved. The dissipation rate for 4th-, 8th- and 16th-order schemes appear to fall directly upon those of the spectral data. Zooming in at the point of peak dissipation, Figure 2(b) shows that 8th- and 16th-order schemes do better match with the reference spectral data.

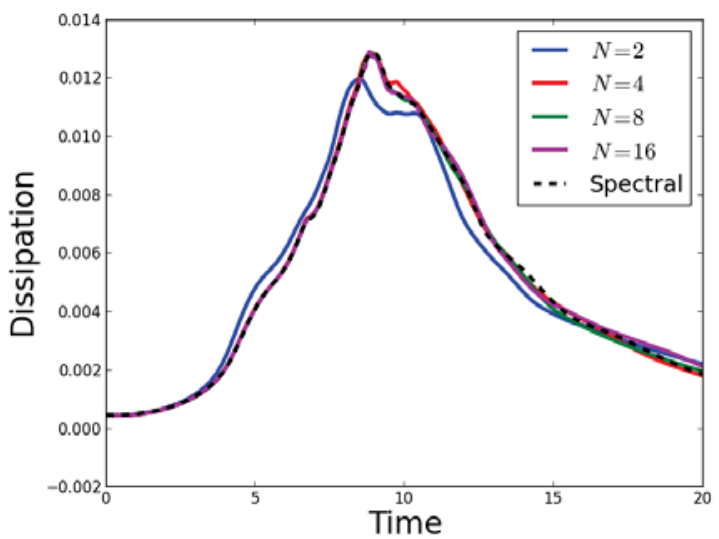

(a) Dissipation

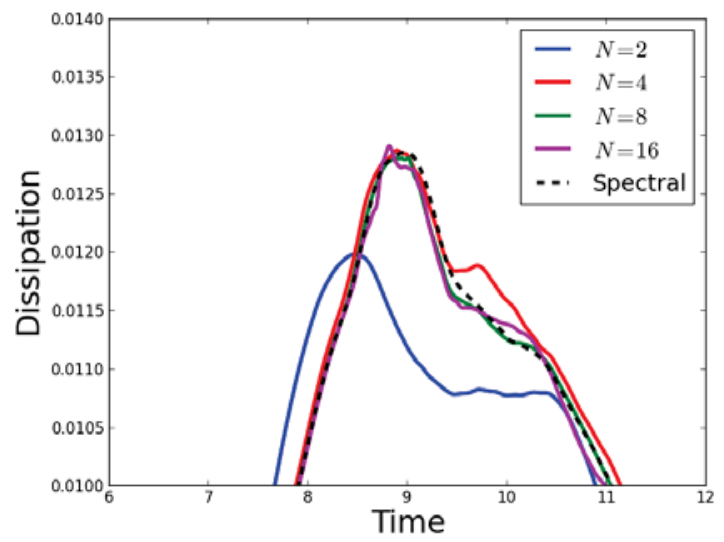

(b) Dissipation (Zoomed)

Figure 2. Taylor-Green vortex problem at $M=0.1, R e=1600$, computed using $256^{3}$ degrees of freedom.

We present further validation of our numerical method by performing direct numerical simulation (DNS) of the Taylor-Green vortex problem at $R e=160$. At this lower Reynolds number, we are able to use fewer degrees of freedom to resolve the flow so that we may observe the asymptotic convergence rate of our numerical scheme. We assess the quality of our numerical solutions by computing individual terms in the kinetic energy evolution equation. For compressible flow, the kinetic energy dissipation rate is given by the sum of three contributions $\epsilon=\epsilon_{1}+\epsilon_{2}+\epsilon_{3}=-\frac{d E_{k}}{d t}$ :

$$
\begin{aligned}
\epsilon_{1} & =\frac{1}{\Omega} \int_{\Omega} 2 \mu s_{i j} s_{i j} d \Omega \\
\epsilon_{2} & =\frac{1}{\Omega} \int_{\Omega} \lambda u_{k, k} u_{k, k} d \Omega \\
\epsilon_{3} & =-\frac{1}{\Omega} \int_{\Omega} p u_{k, k} d \Omega
\end{aligned}
$$

where $s_{i j}=\frac{1}{2}\left(u_{i, j}+u_{j, i}\right)$ is the strain-rate tensor. Since the flow is nearly incompressible, we expect the dissipation due to the bulk viscosity $\left(\epsilon_{2}\right)$ and the pressure-dilatation term $\left(\epsilon_{3}\right)$ to be small. The kinetic energy dissipation rate is then approximately equal to $\epsilon \approx \epsilon_{1}$. However, for the compressible simulation this does not hold exactly. Time histories of $-\frac{d E_{k}}{d t}, \epsilon_{1}, \epsilon_{2}$ and $\epsilon_{3}$, computed using an 8th-order DG scheme in both space and time with 128 degrees of freedom (DOF) in each coordinate direction, are presented in Figure 2. We note that $\epsilon_{1}, \epsilon_{2}$ and $\epsilon_{3}$ must be computed using the "lifted" gradients, accounting for the jumps in the solution between elements, in order to be consistent with our DG discretization. Compressibility effects are evident in oscillations of the pressure dilatation term $\left(\epsilon_{3}\right)$, These oscillations are 3 orders of magnitude less than the viscous dissipation due to strain $\left(\epsilon_{1}\right)$, however they do not go away with further mesh refinement, but correspond to the true compressible result given the isopycnic initial condition. 

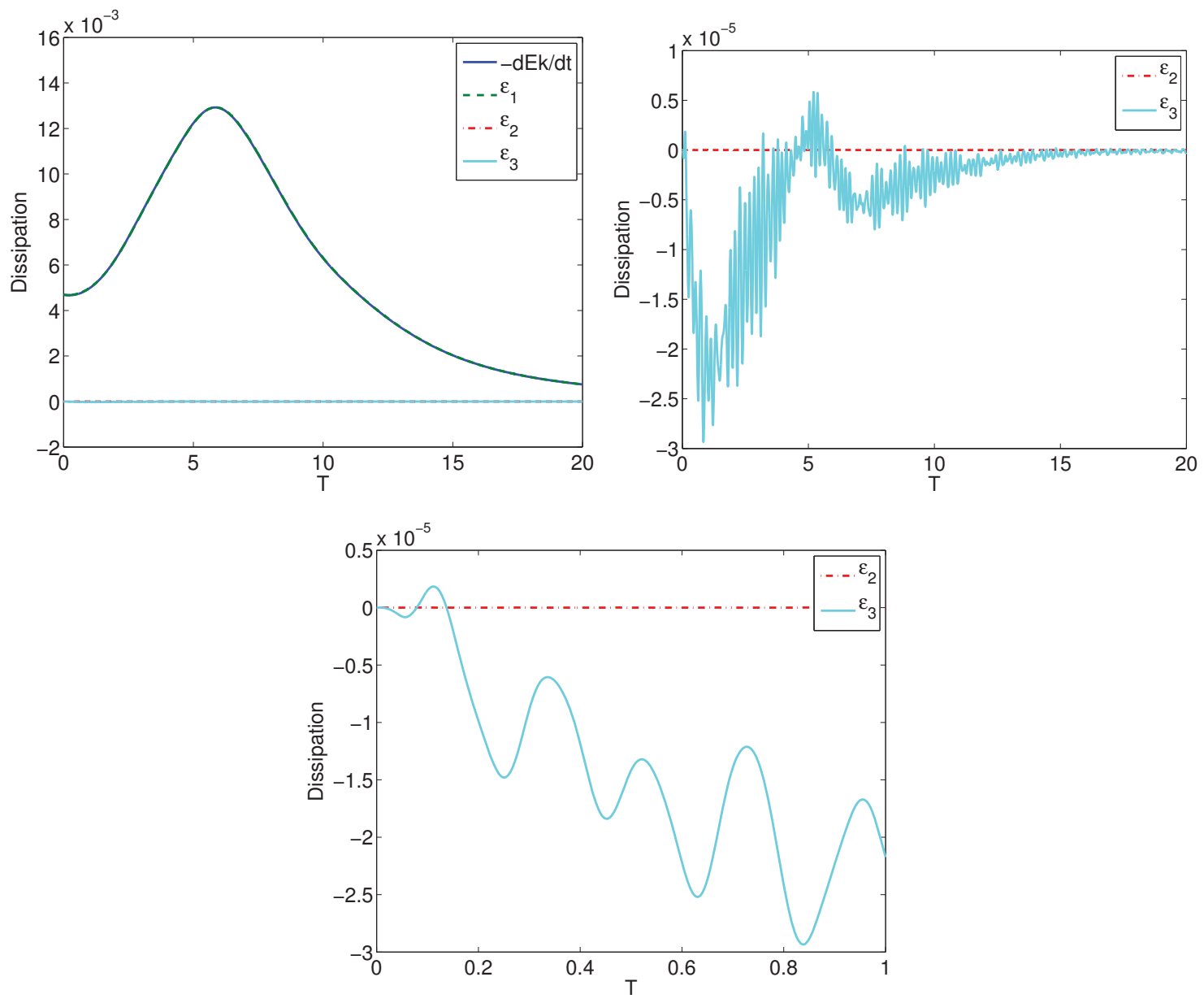

Figure 3. Kinetic energy dissipation balance for the Taylor-Green vortex problem at $M=0.1, R e=160$, computed using $N=8$, and $128^{3}$ degrees of freedom. 
Figure 4 shows the evolution of $-\frac{d E_{k}}{d t}, \epsilon_{1}, \epsilon_{2}$ and $\epsilon_{3}$ computed using a the space-time DG scheme with a 4th-order temporal discretization and 2nd-order spatial discretizations using 48 and 128 degrees of freedom (DOF) in each coordinate direction. The pressure-dilatation, $\epsilon_{3}$, has a significant bias, contributing large net positive kinetic energy dissipation. In particular, at the lower resolution, using 48 DOFs in each direction, the pressure-dilatation contribution to the dissipation corresponds to nearly a third of the total. With 128 DOFs in each direction, the contribution of the pressure-dilatation term is significantly smaller, while the kinetic energy dissipation is predominantly due to the physical dissipation, $\epsilon_{1}$. With increasing mesh refinement, the biased pressure-dilatation term decreases toward zero as shown in Figure 5(a). Alternatively, the biased pressure-dilatation term is reduced with increasing polynomial order as demonstrated in Figure 5(b). With sufficient resolution, the pressure-dilatation term converges to the small (but nonzero) solution presented in Figure 3, which exhibits a damped oscillation to zero. The biased contribution of the pressure-dilatation term appears to be a numerical artifact of the upwind DG scheme, not present in under-resolved simulations using higher-order central-difference schemes. ${ }^{13}$

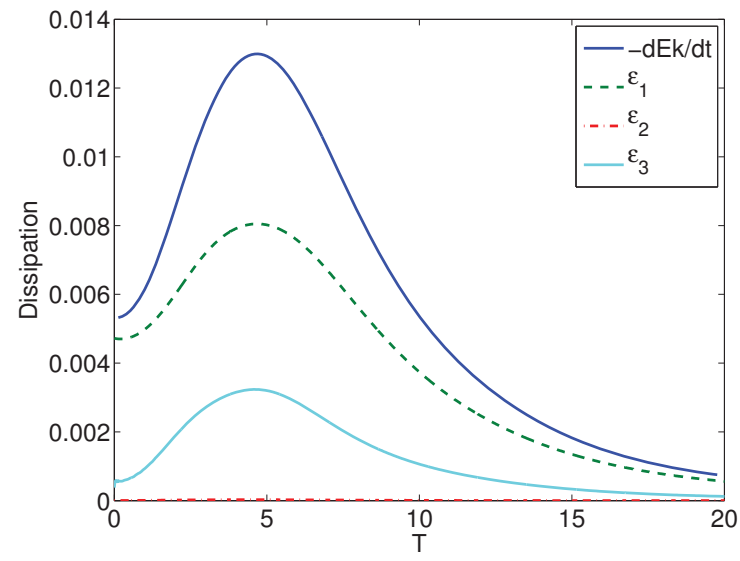

(a) $N=2$ with $48^{3} \mathrm{DOF}$

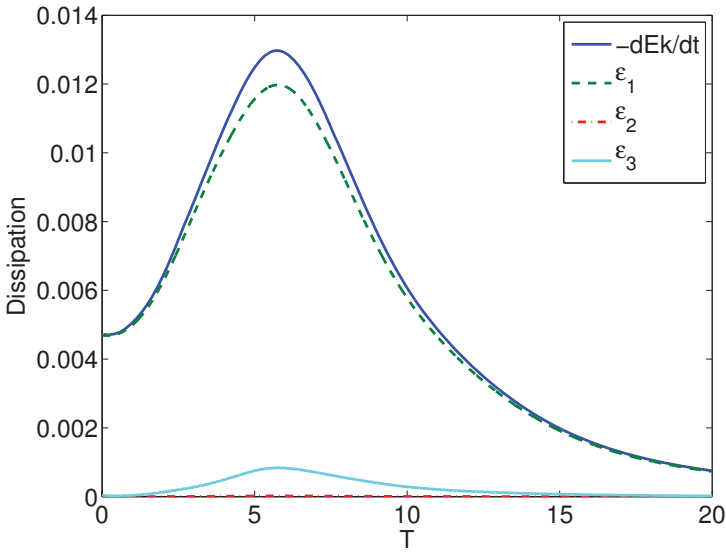

(b) $N=2$ with $128^{3}$ DOF

Figure 4. Kinetic energy dissipation balance for the Taylor-Green vortex problem at $M=0.1, R e=160$, computed using 2nd-order spatial discretization.

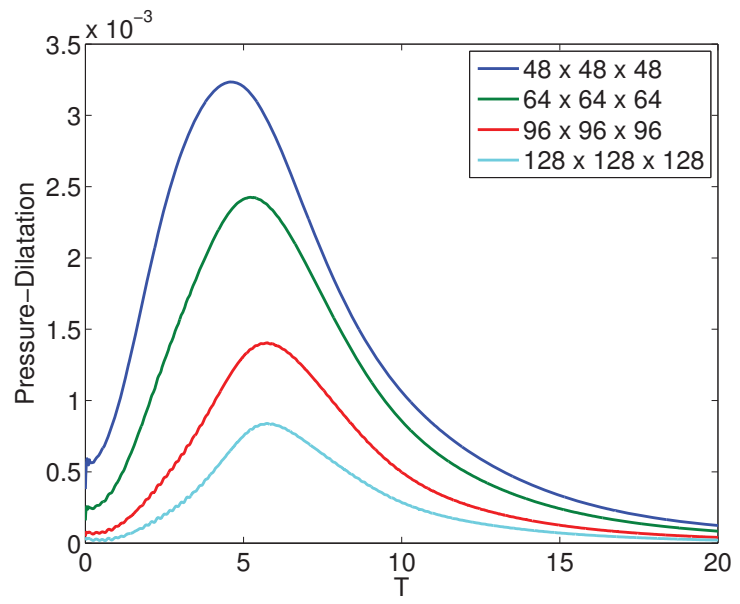

(a) $N=2$

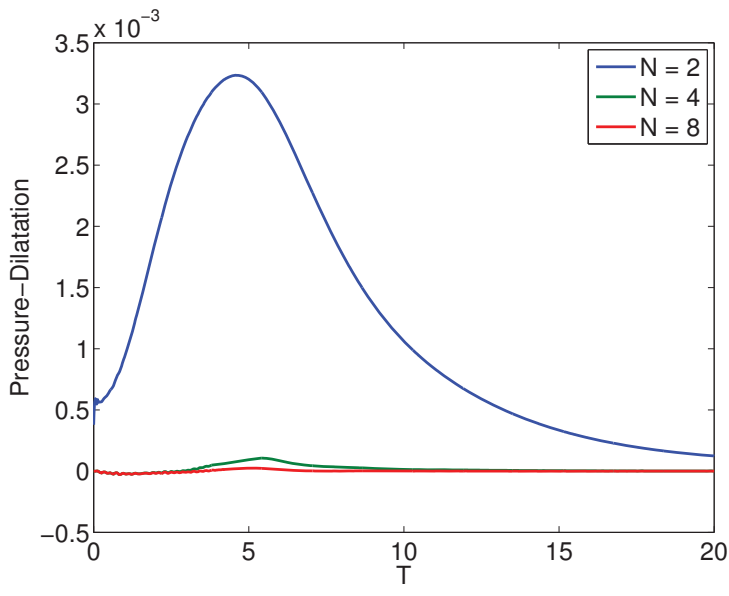

(b) $D O F=48$

Figure 5. Biased pressure-dilatation term for the Taylor-Green vortex problem at $M=0.1, R e=160$, with a) mesh refinement at 2nd-order and b) at constant DOF with increasing polynomial order. 
Our numerical scheme ensures discrete conservation of the state variables: $\rho, \rho u_{i}$ and $\rho E$. However, secondary conservation laws, corresponding to higher-order moments of the conservative states, such as the kinetic energy balance, are not discretely satisfied. The balance of the kinetic energy equation is used as a measure of the presence of numerical errors in the simulation without relying on an exact solution. Specifically, we compute an error which is the difference between the dissipation integrated over the spacetime domain and the change in kinetic energy over the given time-interval. The error in the kinetic energy as a function of the resolution length-scale $h=1 / \mathrm{DOF}^{1 / 3}$ is plotted in Figure 6(a). The 2nd-, 4th- and 8thorder schemes show convergence of the error at slightly better than formal rate. Increasing the polynomial order significantly reduces the error. At the lowest resolution, the 4th-order scheme has an error an order of magnitude less than the 2nd-order scheme, while using the 8th-order scheme with the same number of degrees of freedom gives nearly another order of magnitude reduction in error. The error versus the corresponding CPU time given in terms of TAU benchmark work units ${ }^{6}$ in presented in Figure 6(b). The benefit of the higher order scheme is clearly seen in this plot. The 4 th order scheme on the coarsest mesh is able to attain the same error level as the 2nd-order scheme on the finest mesh, but with a cost which is nearly two orders of magnitude less. Extrapolating the data, the 8th-order scheme at the lowest resolution requires 4 orders of magnitude less work than the 2nd-order scheme to reach a comparable error level.

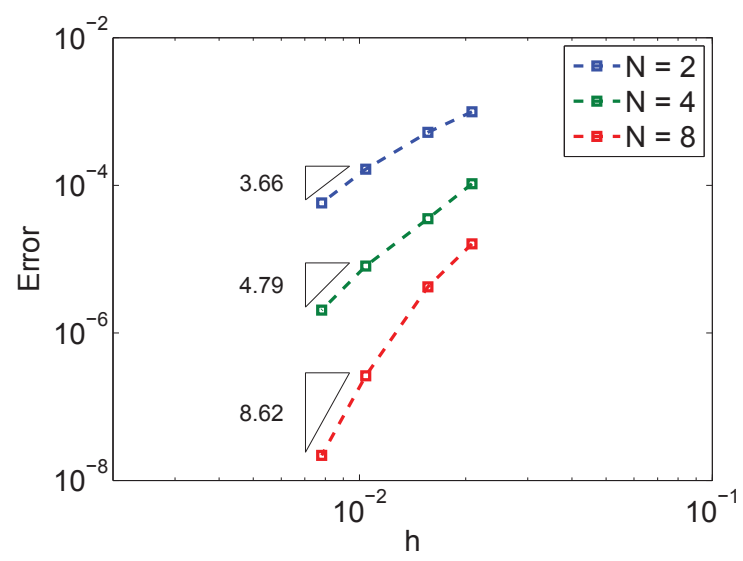

(a) Error vs $h$

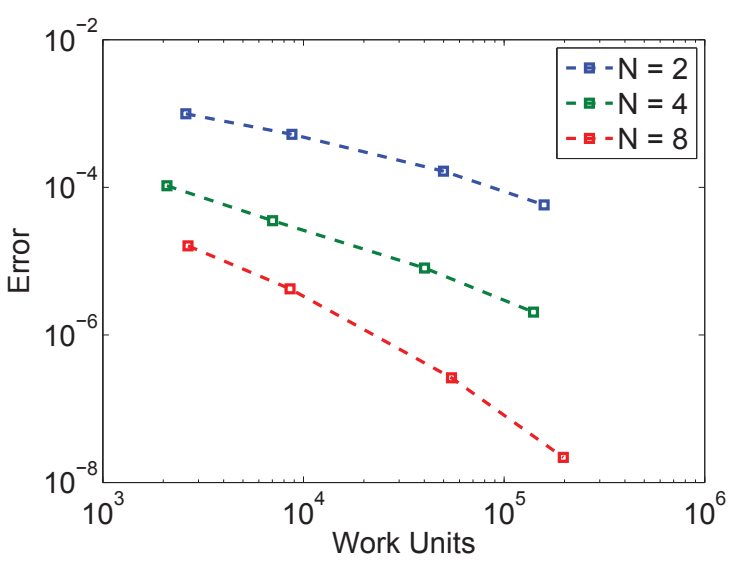

(b) Error vs Work

Figure 6. Error in kinetic energy evolution for Taylor-Green vortex problem at $M=0.1, R e=160$.

\section{Turbulent Channel Flow}

We consider the flow in a channel at $R e_{\tau}=180$, where $R e_{\tau}=\frac{u_{\tau} \delta}{\nu}$ is the Reynolds number based on the wall shear velocity, $u_{\tau}=\sqrt{\tau_{w} / \rho}$, the channel half-width, $\delta$, and the kinematic viscosity, $\nu=\mu / \rho$. This flow has been previously studied using incompressible DNS by Kim et al., ${ }^{14}$ and provides a good validation of our numerical scheme for wall-bounded DNS. The simulations performed are nearly incompressible with a Mach number of approximately 0.1 based on the bulk velocity and mean speed of sound. Following Kim et al. ${ }^{14}$ the domain is of size $4 \pi \delta \times 2 \delta \times 2 \pi \delta$, corresponding to stream-wise, normal, and span-wise directions, respectively. The domain is periodic in the stream-wise and span-wise directions while adiabatic no-slip boundary conditions are applied at the channel walls. The flow is driven by a constant body force applied to the stream-wise momentum equation.

DNS are computed using the space-time DG scheme with a 4th-order temporal discretization and an 8th-order spatial discretization. A mesh refinement study using spatial discretizations with $96 \times 64 \times 80$, $144 \times 96 \times 120,192 \times 128 \times 160$ and $288 \times 192 \times 240$ degrees of freedom in the stream-wise, wall normal and span-wise directions. On the finest mesh, this corresponds to an average spacing in the stream-wise and span-wise directions of $\Delta x^{+} \approx 8$ and $\Delta z^{+} \approx 5$ per DOF. The degrees of freedom are clustered towards the walls such that the first element in the wall normal direction extends to approximately $y^{+}=5$, with average spacing in the first element of $\Delta y^{+} \approx 0.65$. The corresponding reference spectral simulation of Kim et al. 


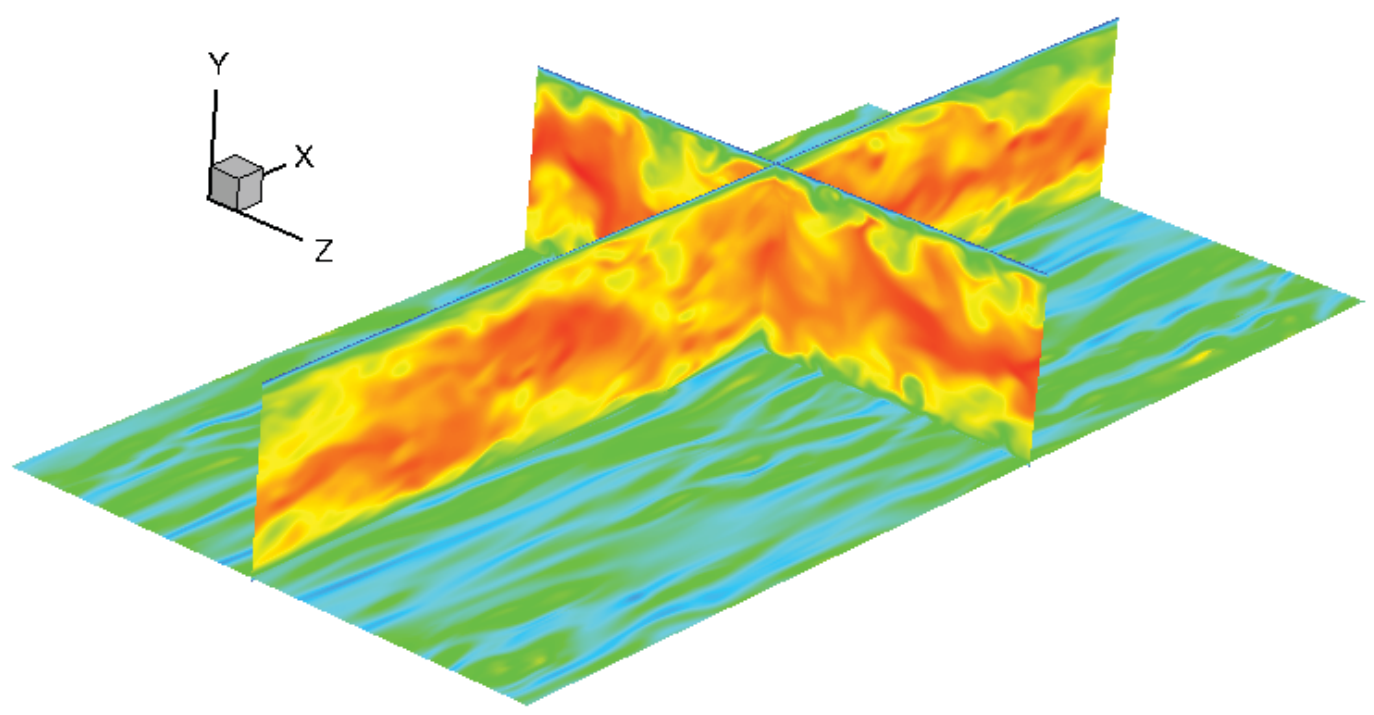

Figure 7. Channel flow at $R e_{\tau}=180$, instantaneous stream-wise velocity. Cross-sections of the full domain of size $4 \pi \delta \times 2 \delta \times 2 \pi \delta$. $x-, y$ - and $z-$ axes correspond to stream-wise, normal and span-wise directions respectively.

used a mesh with $192 \times 128 \times 160$ DOFs.

The flow was initialized to the laminar profile on the coarser meshes and turbulence was induced by applying the forcing technique of Eswaran and Pope. ${ }^{15}$ The forcing is stopped after two eddy turnover times, $h / u_{\tau}$ after which point the turbulence is self-sustaining. The solution was transfered from the coarser meshes and used as the initial condition on the finer meshes. Statistics were collected over 20 eddy turnover times for all but the $288 \times 192 \times 240$ DOF mesh, for which statistics were collected over 10 eddy turnover times. We do not believe that the flow achieved a statistically converged state, however, these simulations are sufficient to provide a validation test case for our numerical scheme.

Figure 7 contains cross-sections of the instantaneous stream-wise velocity on the mesh with $192 \times 128 \times 160$ degrees of freedom. The cross-sections in the stream-wise and span-wise directions show the presence of large eddies on the order of the channel width $2 \delta$. The presence of streak-like structures near the walls may be seen in the cross-section in the wall normal direction. Contours of vorticity magnitude colored by the stream-wise velocity are shown in Figure 8. The presence of vortical structures including hairpin vortices are clearly visible.

The mean velocity, $\bar{u}$, normalized by $u_{\tau}$, using the meshes with 64 and 192 degrees of freedom in the wall normal direction as well as the incompressible DNS data of Kim et al. ${ }^{14}$ are plotted in Figure 9. Even at the lower mesh resolution, the 8th-order DG results show good agreement with the reference DNS results obtained using a spectral method. In particular, we note that the viscous sublayer and buffer layers computed with the DG scheme match exactly with the DNS data despite this being resolved within only two elements in the wall-normal direction on the coarsest mesh. Mismatch of the velocity profile in the core of the flow is likely due to not having reached a statistically converged state. Figure 10 shows the corresponding r.m.s. velocity fluctuations $u_{r m s}=\left(\overline{u^{\prime} u^{\prime}}\right)^{1 / 2}$. The corresponding Reynolds shear stress, $\overline{u^{\prime} v^{\prime}}$ is plotted in Figure 11. The data agrees well with those of Kim et al. with more resolution improving the correlation.

Again, looking at the balance of higher-order moments of the conservative state provides further validation of our numerical scheme. The evolution equation for the Reynolds stresses for incompressible flow are given by:

$$
\frac{D}{D t} \overline{u_{i}^{\prime} u_{j}^{\prime}}=P_{i j}+\epsilon_{i j}+\Phi_{i j}+D_{i j}^{p}+D_{i j}^{t}+D_{i j}^{v}
$$

where $\frac{D}{D t}$ denote the material derivative, while the balance terms on the right-hand side of (18) are given 


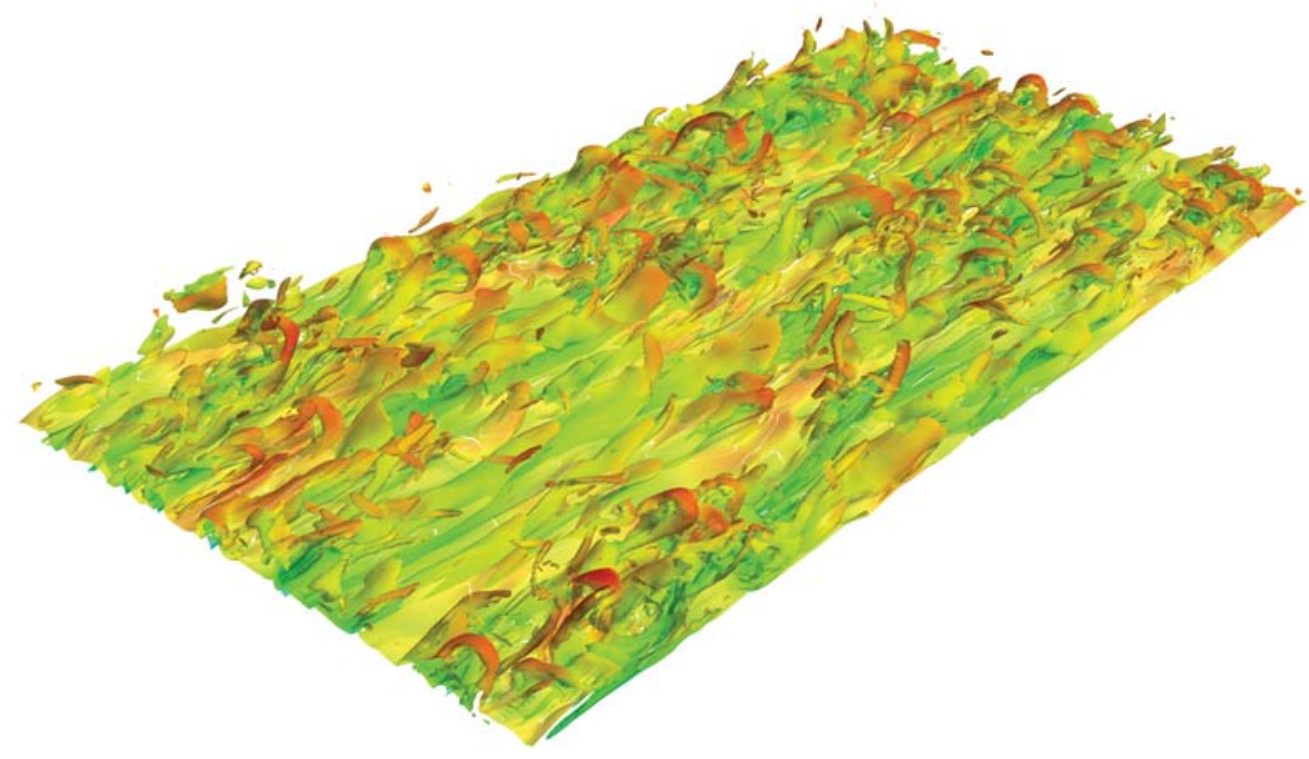

Figure 8. Channel flow at $R e_{\tau}=180$, instantaneous contours of vorticity magnitude colored by stream-wise velocity.

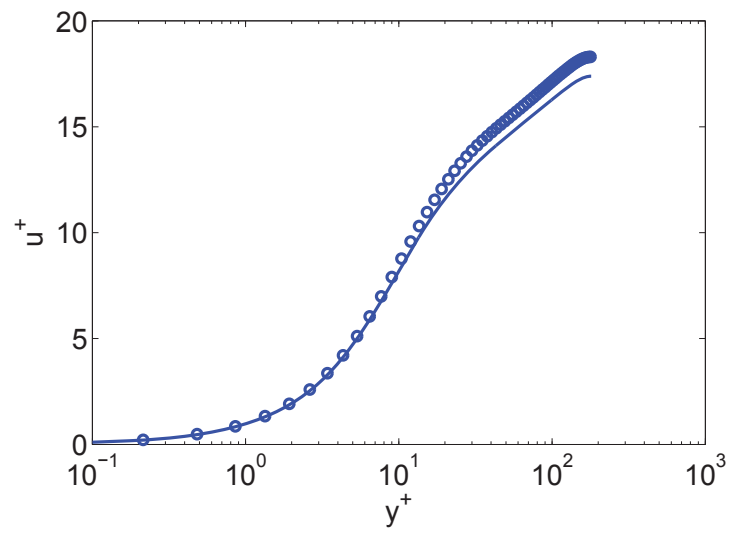

(a) $96 \times 64 \times 80$

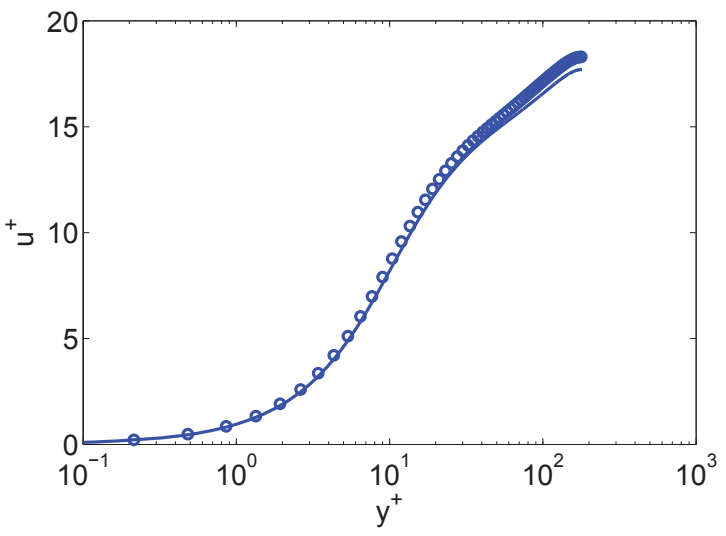

(b) $288 \times 192 \times 160$

Figure 9. Channel flow at $R e_{\tau}=180$, mean velocity profile. (Solid lines are DG solution, symbols are solution of Kim et al. ${ }^{14}$ ) 


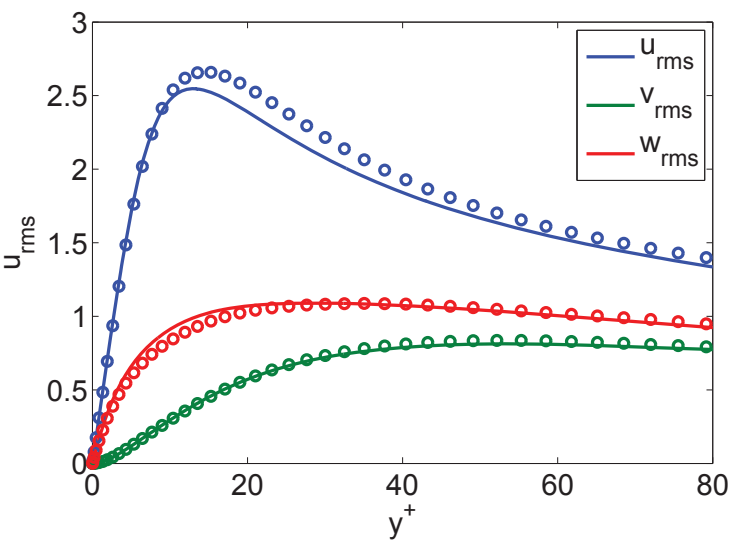

(a) $96 \times 64 \times 80$

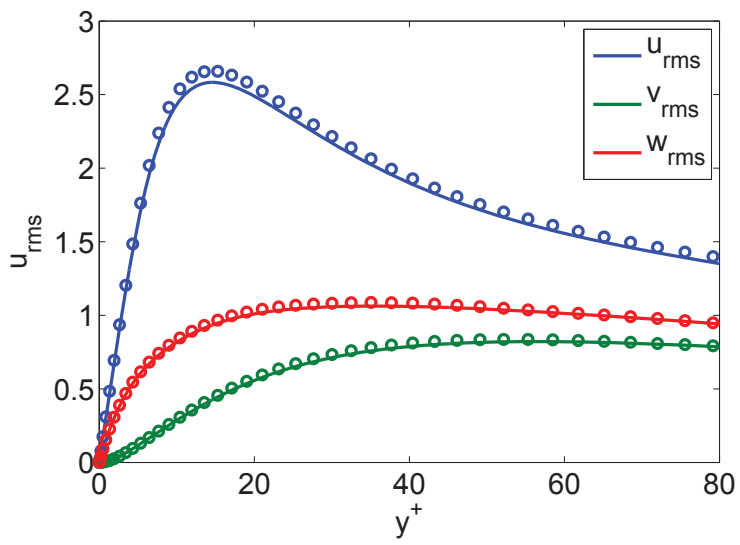

(b) $288 \times 192 \times 240$

Figure 10. Channel flow at $R e_{\tau}=180$, r.m.s velocity fluctuations. (Solid lines are DG solution, symbols are solution of Kim et al. ${ }^{14}$ )

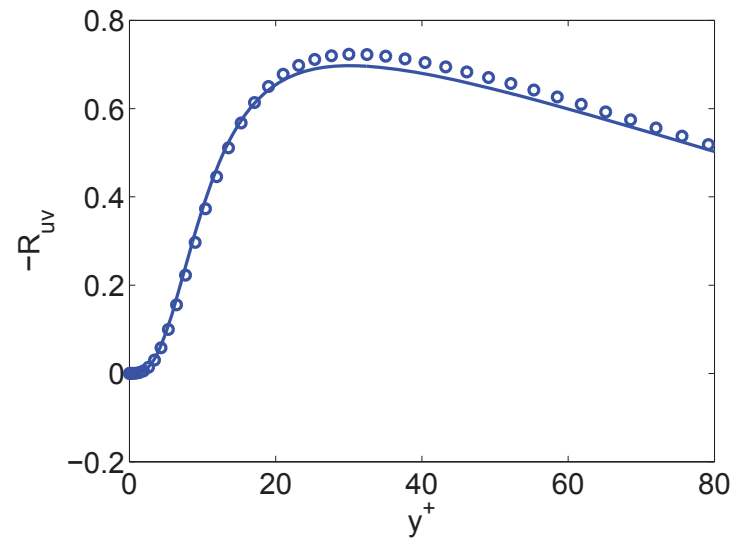

(a) $96 \times 64 \times 80$

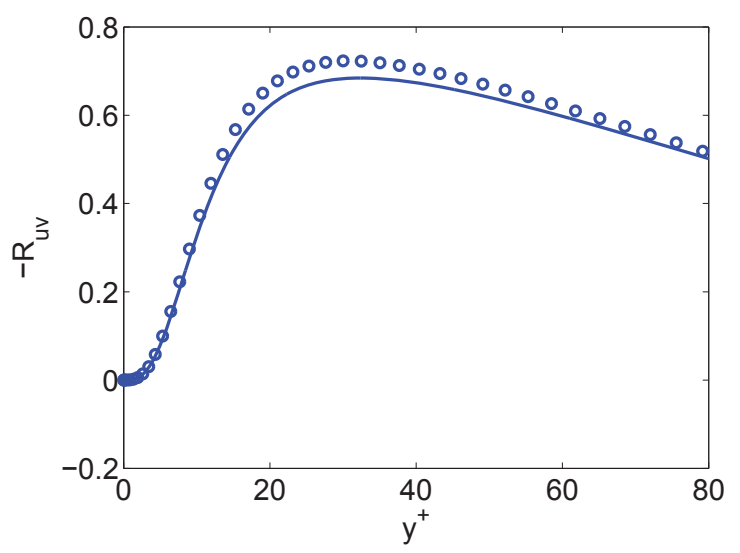

(c) $288 \times 192 \times 240$

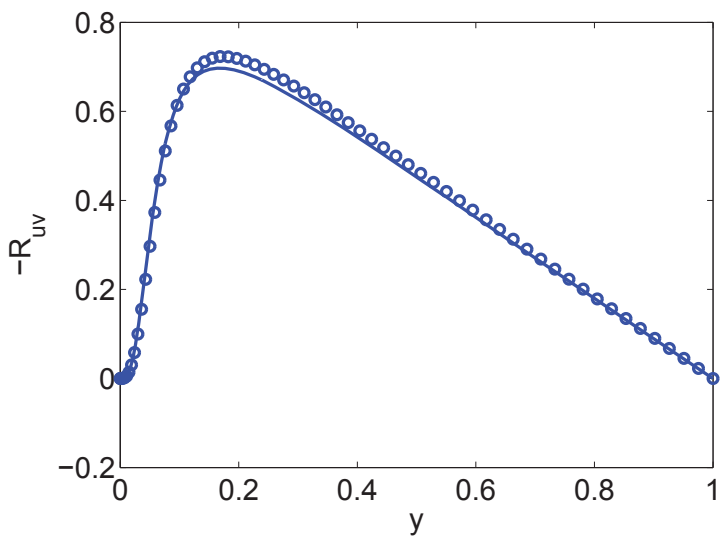

(b) $96 \times 64 \times 80$

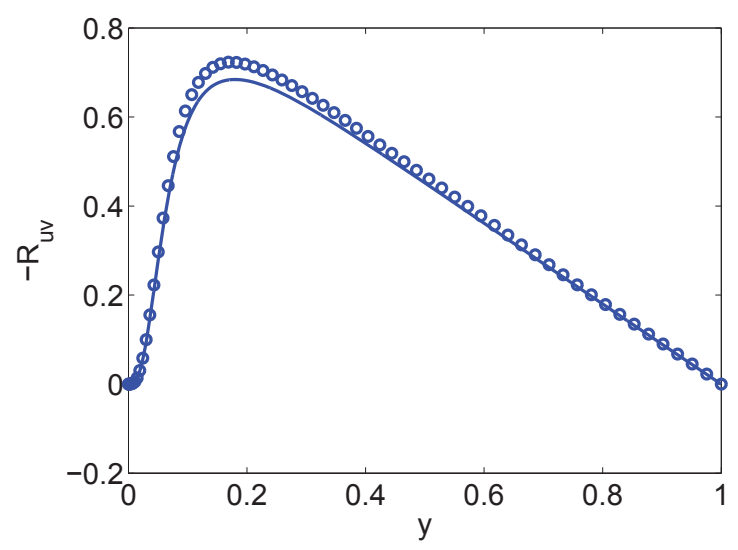

(d) $288 \times 192 \times 240$

Figure 11. Channel flow at $R e_{\tau}=180, R_{u v}$ profile. (Solid lines are DG solution, symbols are solution of Kim et al. ${ }^{14}$ ) 
by:

\begin{tabular}{|c|c|c|c|}
\hline Production & $P_{i j}$ & $=$ & $-\left(\overline{u_{i}^{\prime} u_{k}^{\prime}} \bar{u}_{j, k}+\overline{u_{j}^{\prime} u_{k}^{\prime}} \bar{u}_{i, k}\right)$ \\
\hline Dissipation & $\epsilon_{i j}$ & $=$ & $-2 \nu \overline{u_{i, k}^{\prime} u_{j, k}^{\prime}}$ \\
\hline Pressure Strain & $\Phi_{i j}$ & $=$ & $\overline{p\left(u_{i, j}^{\prime}+u_{j, i}^{\prime}\right)}$ \\
\hline Pressure Diffusion & $D_{i j}^{p}$ & $=$ & $-\left(\overline{p u_{i}^{\prime}} \delta_{j k}+\overline{p u_{j}^{\prime}} \delta_{i k}\right)$ \\
\hline Turbulent Diffusion & $D_{i j}^{t}$ & $=$ & $-\overline{\left(u_{i}^{\prime} u_{j}^{\prime} u_{k}^{\prime}\right)_{, k}}$ \\
\hline Viscous Diffusion & $D_{i j}^{v}$ & $=$ & $\left(\nu\left(\overline{u_{i}^{\prime} u_{j}^{\prime}}\right)_{, k}\right)$ \\
\hline
\end{tabular}

We compute the incompressible Reynolds stresses and balance terms in order to compare with previous simulations of Kim et al. ${ }^{14}$ For this low Mach number flow, the neglected compressible terms are small. Figure 12 contains the terms in the evolution of the Reynolds shear stress $\overline{u^{\prime} v^{\prime}}$, normalized by $u_{\tau}$, with increasing mesh resolution. Overall, the profiles obtained on each mesh are very similar. However, on the coarsest mesh large oscillations appear in the pressure-strain and pressure-diffusion terms at the interface between elements close to the wall. With increasing mesh resolution these oscillations go away and there is excellent agreement with the reference spectral data. We postulate that the large oscillations are due to insufficient resolution resulting in non-physical pressure-strain/pressure-diffusion as seen at low resolution in the Taylor-Green vortex test case. When present, these numerical artifacts serve as an indication of a lack of resolution.

The statistical distributions of the components of the velocity near the point of peak Reynolds shear stress are presented in Figure 13. The stream-wise velocity, $u$, has an obviously non-Gaussian profile with skewness of -0.74 and flatness of 3.50. The wall-normal velocity component, $v$, deviates somewhat from the Gaussian distribution with skewness of 0.28 and flatness of 3.43, while the $w$ has a nearly Gaussian distribution with skewness of 0.006 and flatness of 3.26. In the following section we will compare these baseline distributions with those seen in different regions of the flow about the periodic hill.

\section{2D Periodic Hill}

The 2D periodic hill problem has been widely studied as a model problem for separated turbulent flow. ${ }^{2-5}$ The geometry we consider was presented by Mellen et al. ${ }^{2}$ and has been used as a test case in several studies (c.f. Breuer et al. ${ }^{5}$ and references therein). The geometry consists of a periodic channel with a 2D hill restriction. The size of the domain is $9 h \times 3.035 h \times 4.5 h$ in the stream-wise, wall-normal and span-wise directions, where $h$ is the height of the hill. The flow is driven by a constant body force to ensure a given mass flux.

We consider the flow at a Reynolds number $R e=10,595$, where the Reynolds number is defined using the bulk velocity and the height of the channel above the hill. Figure 14 shows the mean velocity profile, $\bar{u}$, and the r.m.s. velocity, $u_{r m s}=\left(\overline{u^{\prime} u^{\prime}}\right)^{1 / 2}$. This flow has several complicated features which pose modeling challenges. The flow accelerates up the windward side of the hill, separates over the top and a large recirculation region develops on the leeward side of the hill. Reattachment occurs on the flat surface between successive hills. Figure 15 shows the instantaneous isocontour corresponding to zero stream-wise velocity colored by entropy, which highlights the extent of the reversible flow region. Modeling challenges involve accurately predicting the point of separation, which can have significant impact on the size of the recirculation bubble. ${ }^{5}$ Previous LES at $R e=10,595$ have shown the presence of small recirculation regions near the top of the hill and at the base of the hill on the windward side. ${ }^{3}$ These studies have also noted large span-wise velocity fluctuations beyond the reattachment point due to large-scale eddies convecting downstream from the shear layer. ${ }^{3}$ This phenomenon dictates that significant resolution is needed near the shear layer.

Simulations were performed at $R e=10,595$ using our space-time DG formulation with a 4th-order temporal discretization and an 8th-order spatial discretization. We have used a sequence of meshes using $128 \times 64 \times 64,192 \times 96 \times 96,256 \times 128 \times 128$ and $384 \times 192 \times 192$ degrees of freedom in stream-wise, wall-normal and span-wise directions. At each mesh resolution 8th-order polynomial curvilinear meshes were generated to match the solution order by defining the location of the Gauss-Legendre-Lobatto nodes for each element using a pseudo-spectral rational interpolation from an underlying structured mesh. The cross-section of the 




(a) $96 \times 64 \times 80$

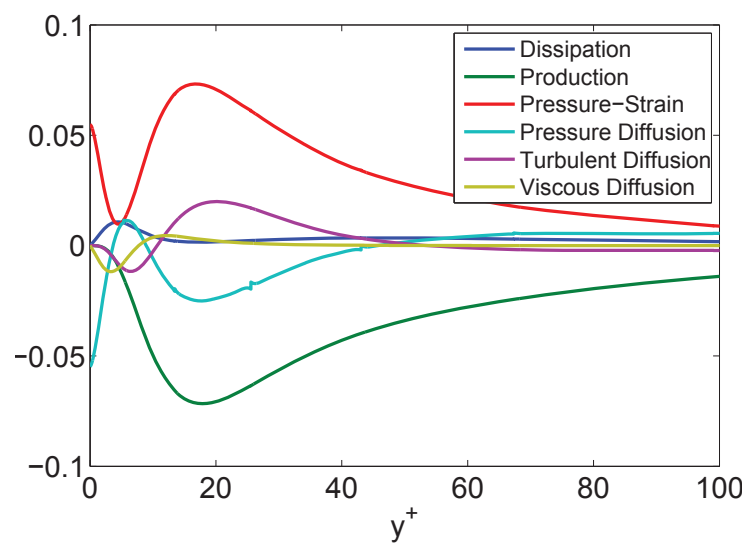

(c) $192 \times 128 \times 160$

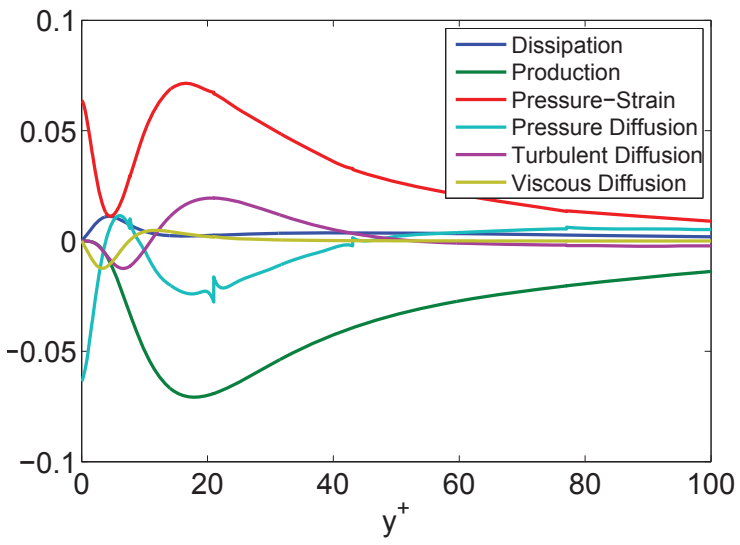

(b) $144 \times 96 \times 120$

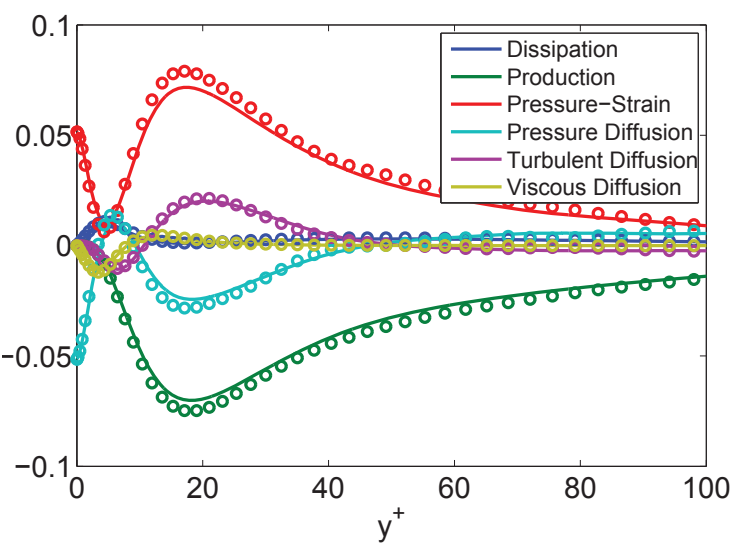

(d) $288 \times 192 \times 240$

Figure 12. Channel flow at $R e_{\tau}=180$, balance terms in evolution of Reynolds shear stress. (Solid lines are DG solution, symbols are solution of $\mathrm{Kim}$ et al. ${ }^{14}$ )
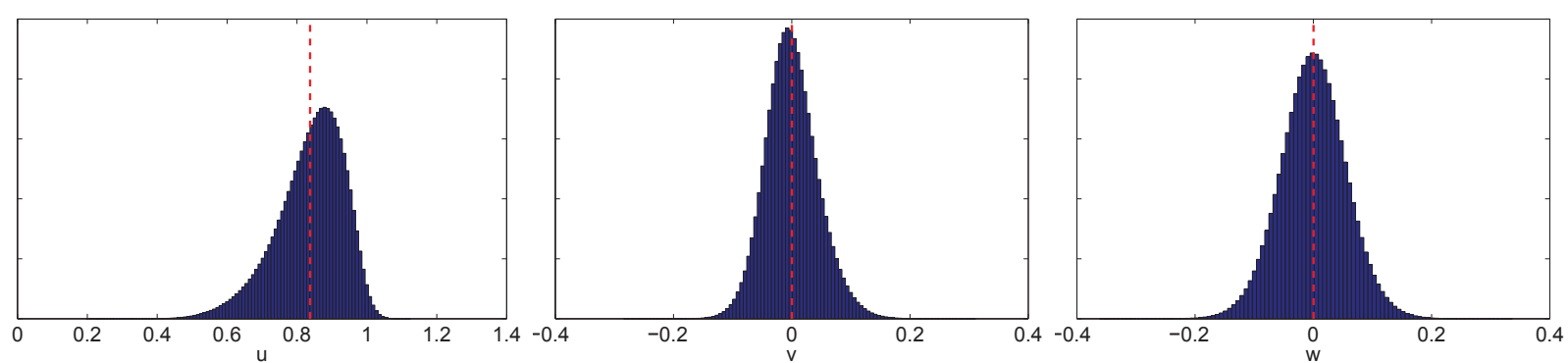

Figure 13. Channel flow at $R e_{\tau}=180$, distribution of velocity components. 


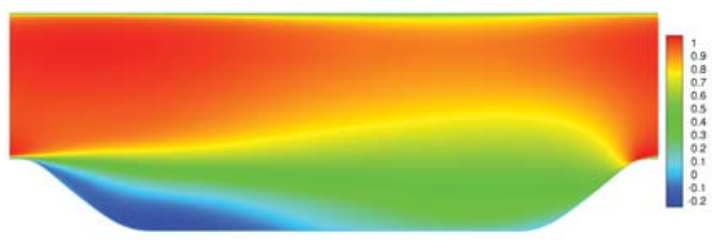

(a) $\bar{u}$

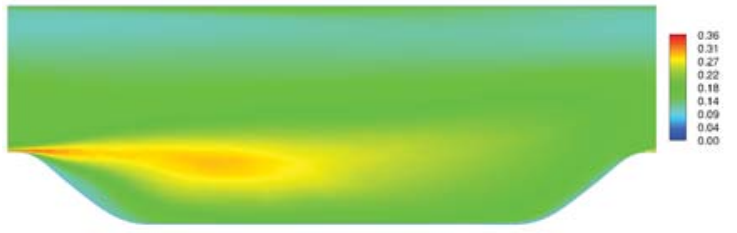

(b) $u_{r m s}$

Figure 14. Mean stream-wise velocity, $\bar{u}$ and r.m.s. stream-wise velocity, $u_{r m s}=\left(\overline{u^{\prime} u^{\prime}}\right)^{1 / 2}$ for Periodic Hill at $R e=10,595$.

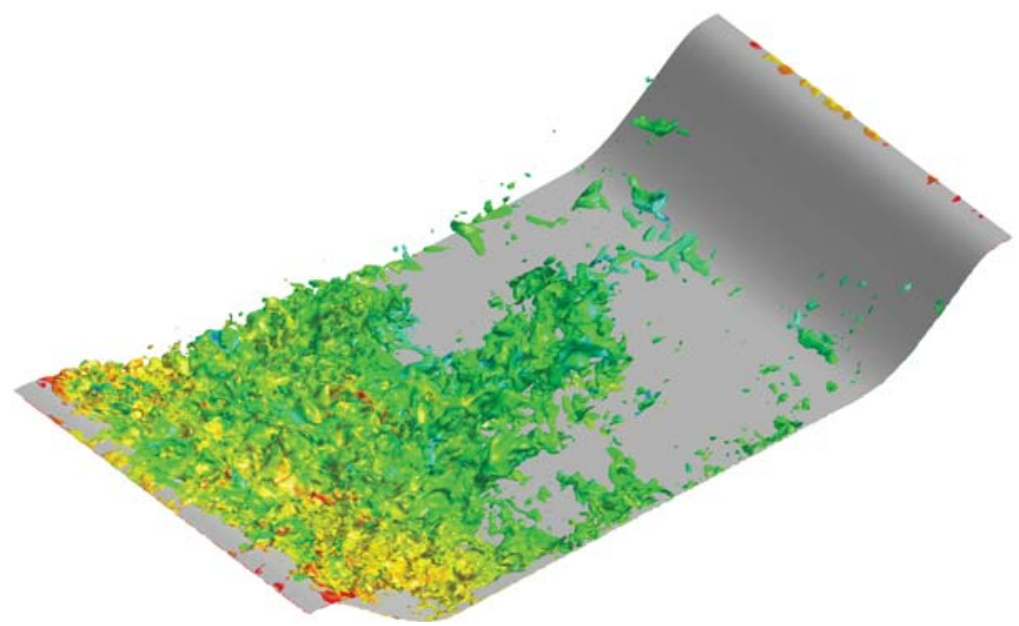

Figure 15. Isocontour of $u=0$ colored by entropy for Periodic Hill at $R e=10,595$. 
mesh consisting of $16 \times 8 \times 8$ elements, corresponding to the simulation using $128 \times 64 \times 64 \mathrm{DOFs}$, is plotted in Figure 16.

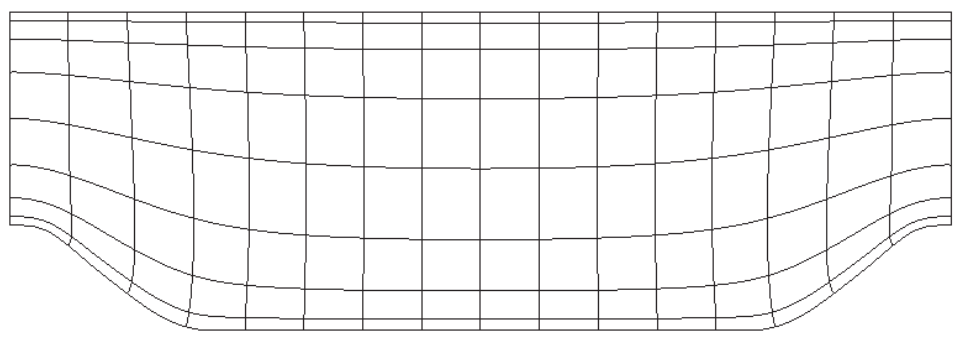

Figure 16. Curvilinear mesh for Periodic Hill.

The simulations were run for approximately 25 flow through periods. We do not believe this is a sufficiently long time to achieve a statistical convergence, however, these simulations serve as an initial validation of our numerical scheme. As a reference, previous LES simulations of this flow by Fröhlich et al. ${ }^{3}$ and Breuer et al. ${ }^{5}$ collected statistics over 55 and 140 flow through times, respectively.

The mean pressure and wall shear stress on the lower surface of the channel computed on the finest mesh is presented in Figure 17. The mean shear stress profile shows the primary separation point at approximately $x=0.20$, with reattachment at $x=4.37$. This matches well with values of $x=0.19$ and $x=4.69$, respectively, for the separation and reattachment points reported by Breuer et al. ${ }^{5}$ Table $\mathrm{V}$ gives the mean separation and reattachment points with increasing mesh resolution. The coarse simulations predict a later separation point and earlier reattachment point, under-predicting the size of the separation bubble. With mesh refinement the DG simulation results converge to the previously reported data. Detailed analysis of the mean shear stress profiles reveals two additional separation points, corresponding to recirculation bubbles at the base of the hill on the wind-ward side and at the peak of the hill. The existence of these two smaller recirculation bubbles is consistent with previous observations by Breuer et al. ${ }^{5}$

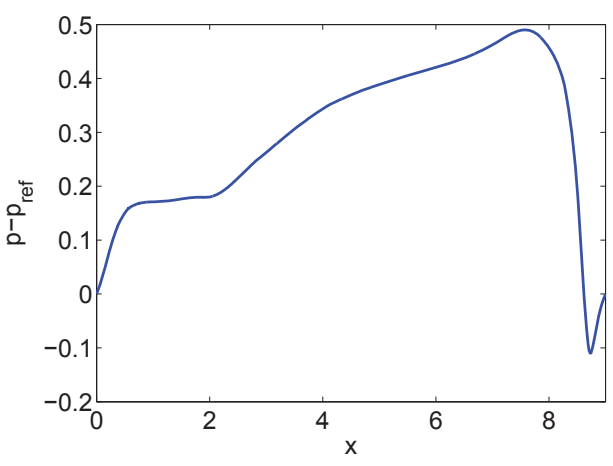

(a) Mean Pressure



(b) Mean Wall Shear Stress

Figure 17. Mean pressure and wall shear stress on lower surface for the periodic hill at $R e=10,595$.

We compare our numerical simulations with LES data of Fröhlich et al. ${ }^{3}$ Figure 18 contains the mean velocity and Reynolds stress profiles computed using the finest mesh resolution at stations with $x \approx 0.05,2.0,4.0$, and 6.0. In the present results, we have plotted the profiles corresponding to data along grid lines, as opposed to constant $x$ stations, in order to simplify our post-processing. Figure 18 shows good agreement between the present simulations and that of Fröhlich et al. Some discrepancies between the computed velocity profiles are attributable to a mismatch in the exact location of the plotted data. The small difference in the size of the separation bubble computed in our simulation and that of Fröhlich et al. ${ }^{3}$ is evident in the velocity profiles at $x \approx 4$. The Reynolds stress profiles obtained with the DG scheme also 


\begin{tabular}{ccc}
\hline Mesh & Separation Point & Reattachment Point \\
\hline $128 \times 64 \times 64$ & 0.28 & 3.94 \\
$192 \times 96 \times 96$ & 0.22 & 4.13 \\
$256 \times 128 \times 128$ & 0.21 & 4.22 \\
$384 \times 192 \times 192$ & 0.20 & 4.37 \\
\hline Breuer et al. ${ }^{5}$ & 0.19 & 4.69 \\
\hline
\end{tabular}

show good agreement with the reference data. However, numerical artifacts are apparent in the profiles, which may be due to insufficient statistical convergence. The Reynolds stress profile at $x=0.05$ shows large values of cross-stream Reynolds stress, $\overline{w^{\prime} w^{\prime}}$. This "splatting", corresponding to turbulent kinetic energy being transfered from stream-wise and wall-normal fluctuations to span-wise fluctuations, was previously described by Fröhlich et al. ${ }^{3}$

The corresponding balance terms in the evolution equation for turbulent kinetic energy, $k=\frac{1}{2} \overline{u_{i}^{\prime} u_{i}^{\prime}}$, are plotted in Figure 19. As with the Reynolds stresses, the computed statistics show good agreement with those of Fröhlich et al. ${ }^{3}$ However, numerical artifacts are apparent in the profiles at the boundary of elements. Given similar behavior in under-resolved turbulent channel flow simulations, this suggests that even the finest mesh simulation is somewhat under-resolved.

We compute the probability distribution function of the velocity at five selected points in the domain. The location of the selected points are plotted in Figure 20, while corresponding distribution of the components of the velocity at each point are presented in Figure 21. Point 1 is selected near the upper surface of the channel, away from the hill and is intended as a baseline profile which is similar to the result presented for the channel flow. Point 2 is selected over the top of the hill. The presence of "splatting" is observed in the long tails of the span-wise velocity distribution relative to the stream-wise and wall-normal velocity distributions. At point 3 in the recirculation region, we see mean large negative $u$ and positive $v$ with distributions which have tails not significantly longer than the baseline. Points 4 and 5 are in the most energetic part of the domain, straddling the mean separation line. At these two points, we see very long tails in the $u$ - and $v$-profiles, corresponding to the large-scale unsteadiness as a result of the separated flow. The skewness of both $u$ and $v$ switch sign, showing that they are straddling the shear layer.

We examine the correlation between the mean strain and Reynolds stresses in order to evaluate the validity of the Boussinesq hypothesis:

$$
-\overline{u_{i}^{\prime} u_{j}^{\prime}} \approx 2 \nu_{t} s_{i j}-\frac{1}{3} \overline{u_{k}^{\prime} u_{k}^{\prime}} \delta_{i j}
$$

The contours of the mean strain and the deviatoric part of the Reynolds stresses are given in Figure 22. In general, regions of large strain are correlated with regions of large Reynolds stress, however the sign of the constant of proportionality in the shear layer changes between the normal and shear stresses. Additionally, in the shear layer, the magnitude of the normal strains are similar to the shear strain, unlike an attached boundary layer where the magnitude of the normal strains typically is much smaller. Coming over the top of the hill, the mean normal strain changes sign while this is not seen in the normal Reynolds stresses. Further, at the rise to the hill, a large normal stress is present in a region with no corresponding strain. Hence, a linear eddy viscosity model will over-predict strongly the turbulent diffusion in this region. These observations are indicative of the insight that can be gained from these simulations to improve RANS modeling.

\section{Summary and Conclusions}

In this paper we have performed direct numerical simulations of turbulent compressible flows at low mach number using a higher-order space-time discontinuous-Galerkin method. We validated our numerical scheme for turbulent flow by studying the Taylor-Green vortex problem. We demonstrated that the upwind DG scheme gives a net positive bias in the pressure-strain contribution to the kinetic energy dissipation for underresolved simulations. This biased contribution disappears with increased resolution and we demonstrated formal error convergence rates up to 8th-order.

Next, we validated our numerical scheme for wall-bounded turbulent flows by performing DNS of turbulent channel flow at $R e_{\tau}=180$. We presented results for mean flow and higher moment quantities which 


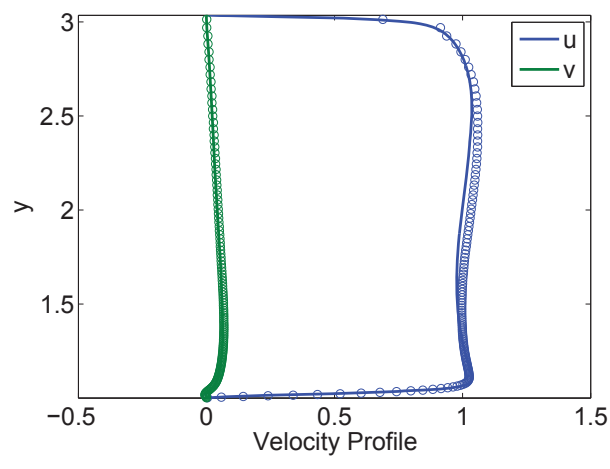

(a) Velocity Profile, $x=0.05$

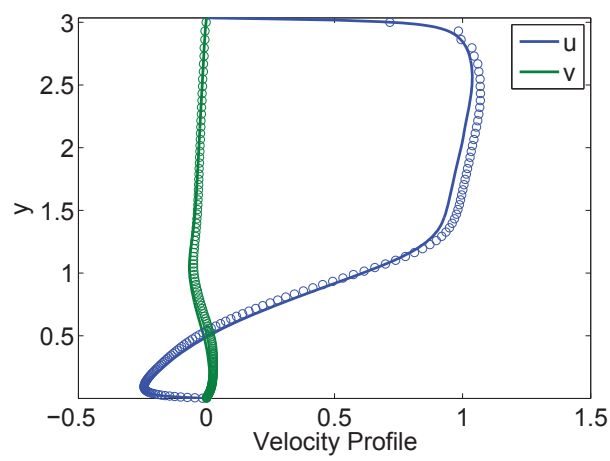

(c) Velocity Profile, $x=2.0$

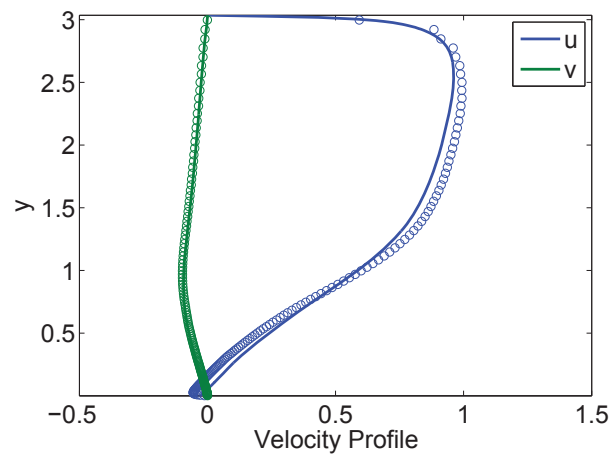

(e) Velocity Profile, $x=4.0$

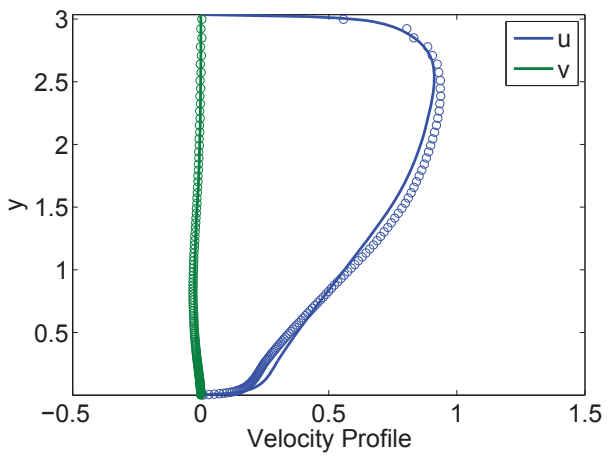

(g) Velocity Profile, $x=6.0$

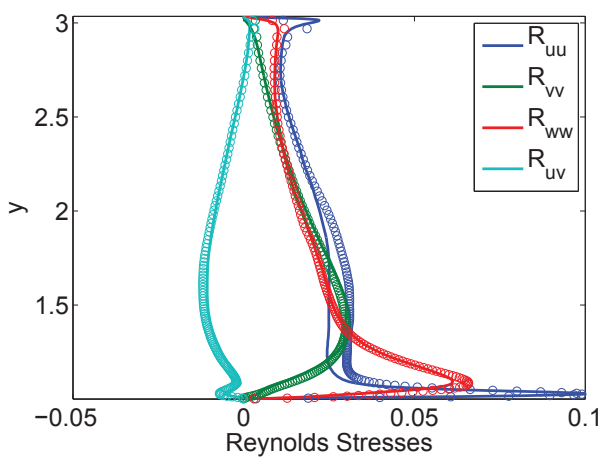

(b) Reynolds Stress, $x=0.05$

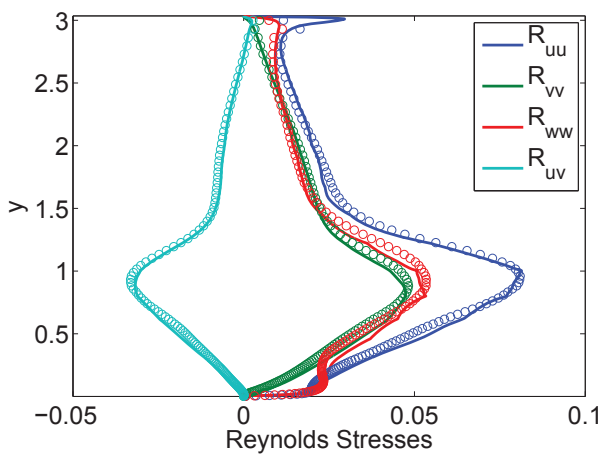

(d) Reynolds Stress, $x=2.0$

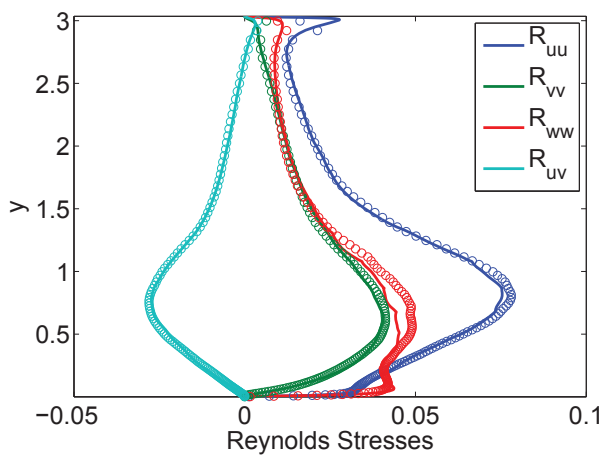

(f) Reynolds Stress, $x=4.0$

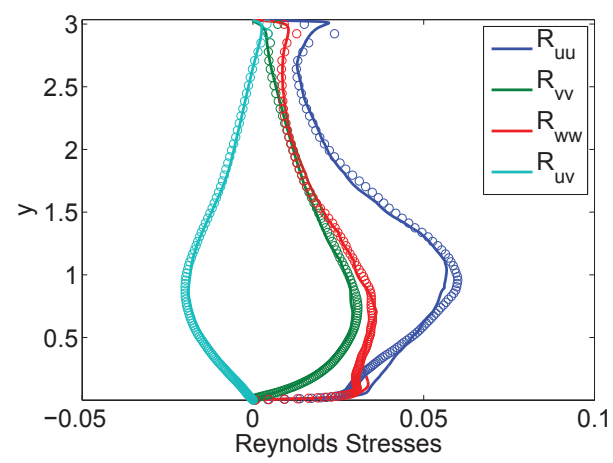

(h) Reynolds Stress, $x=6.0$

Figure 18. Velocity and Reynolds stress profiles at various stations in the domain for the periodic hill at $R e=10,595$. (Solid lines are DG solution, symbols are solution of Fröhlich et al. ${ }^{3}$ ) 


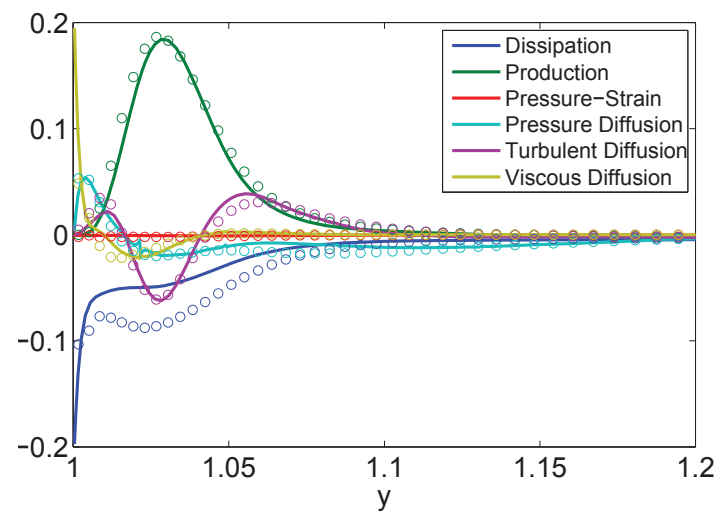

(a) $x=0.05$

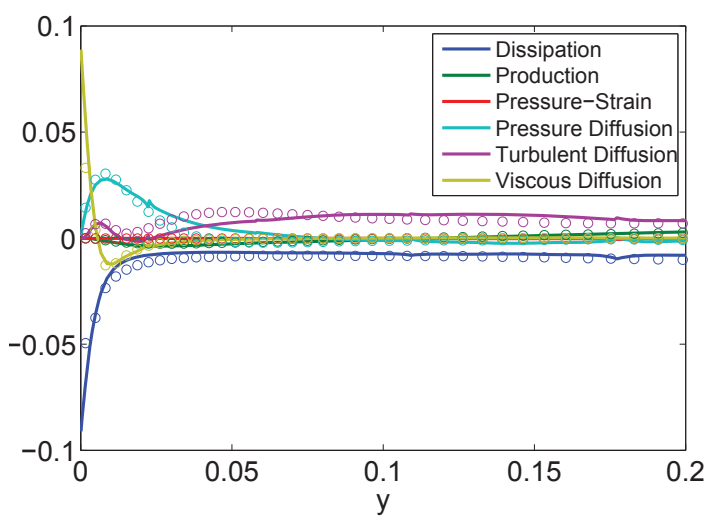

(c) $x=4.0$

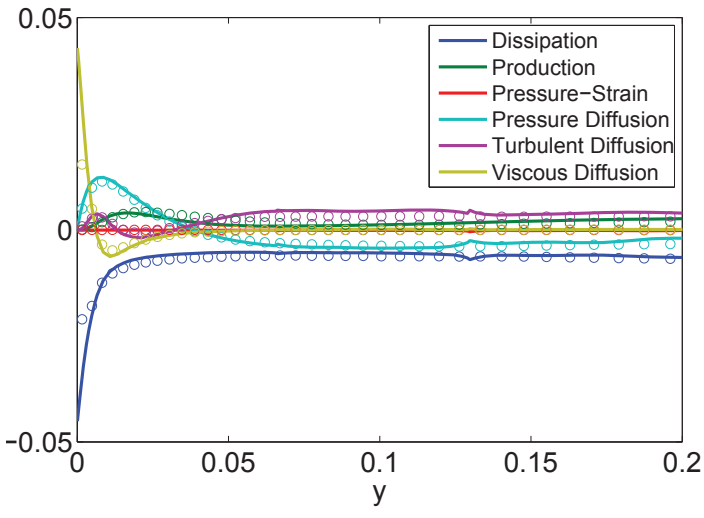

(b) $x=2.0$



(d) $x=6.0$

Figure 19. Balance terms in evolution equation for turbulent kinetic energy at various stations in the domain for the periodic hill at $R e=$ 10,595. (Solid lines are DG solution, symbols are solution of Fröhlich et al. ${ }^{3}$ )

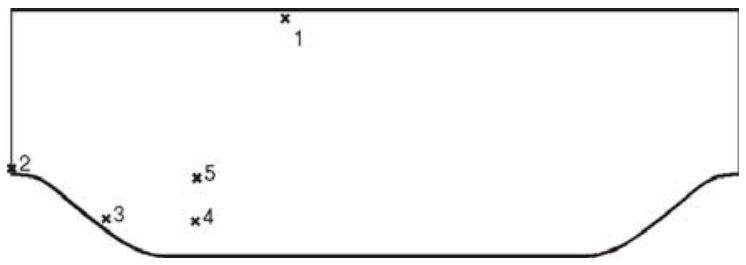

Figure 20. Location of points for computing velocity distributions on the periodic hill at $R e=10,595$ 


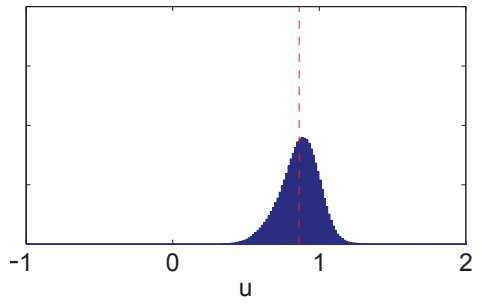

(a) $u$ at point $1:(-0.35,3.29)$

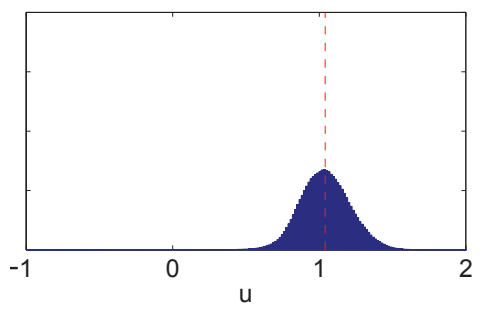

(d) $u$ at point 2 : $(-0.12,4.23)$

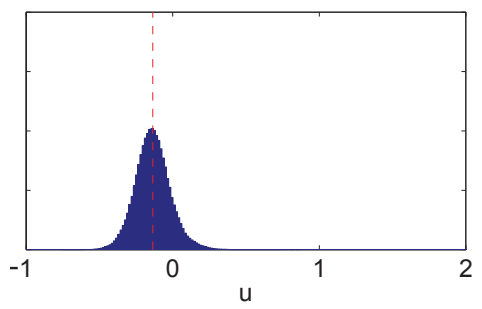

(g) $u$ at point 3: $(0.25,3.70)$

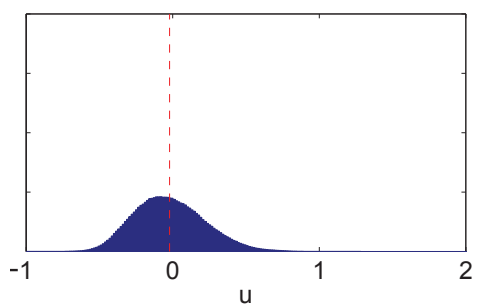

(j) $u$ at point $4:(0.33,3.00)$

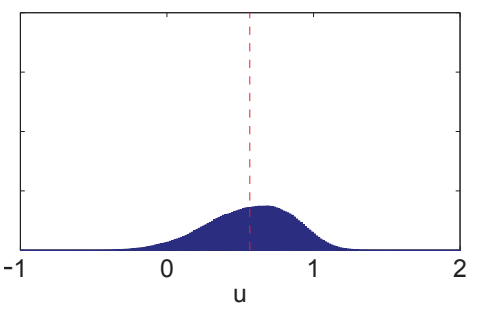

(m) $u$ at point $5:(-0.31,2.79)$

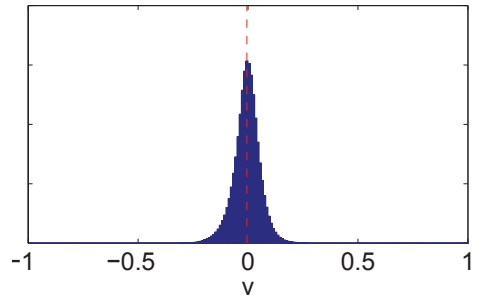

(b) $v$ at point $1:(-0.24,4.81)$



(e) $v$ at point $2:(-0.18,4.01)$

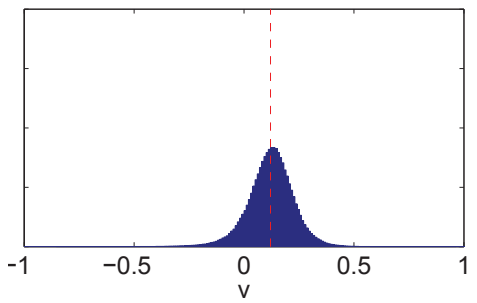

(h) $v$ at point $3:(-0.43,5.72)$

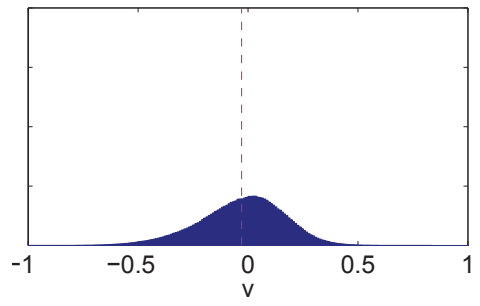

(k) $v$ at point $4:(-0.38,3.31)$

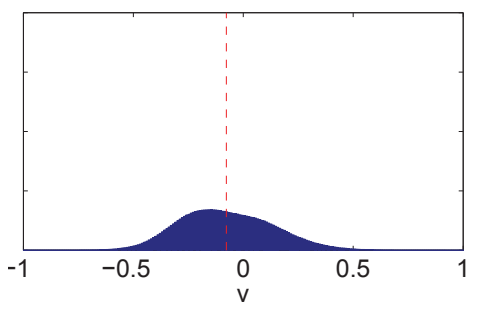

(n) $v$ at point $5:(0.23,2.84)$

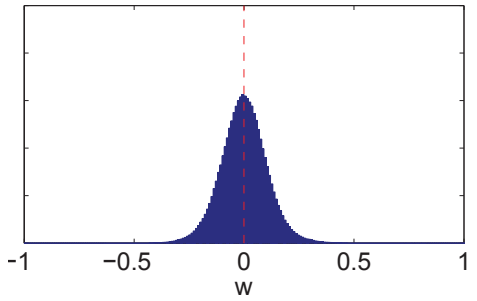

(c) $w$ at point $1:(0.07,3.73)$

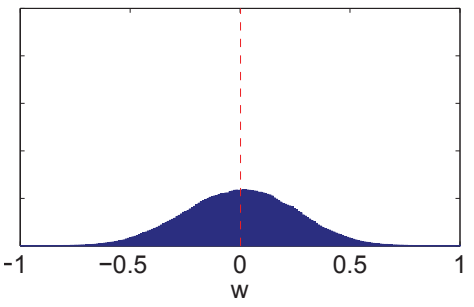

(f) $w$ at point 2: $(-0.03,3.00)$



(i) $w$ at point $3:(-0.13,3.5)$

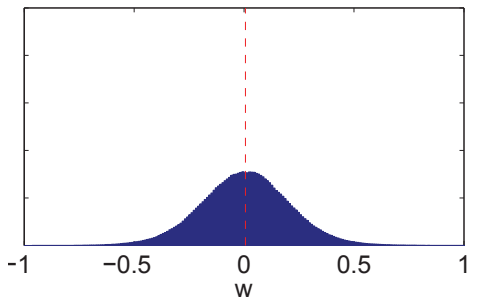

(l) $w$ at point $4:(0.01,3.40)$

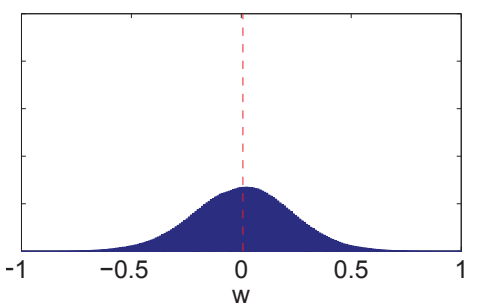

(o) $w$ at point $5:(-0.02,3.21)$

Figure 21. Velocity distributions at selected points for the periodic hill at $R e=10,595$. (Values in parenthesis correspond to skewness and flatness). 


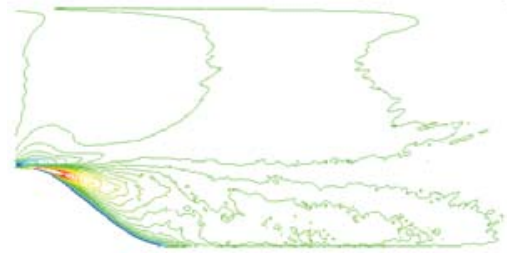

(a) $s_{11}$

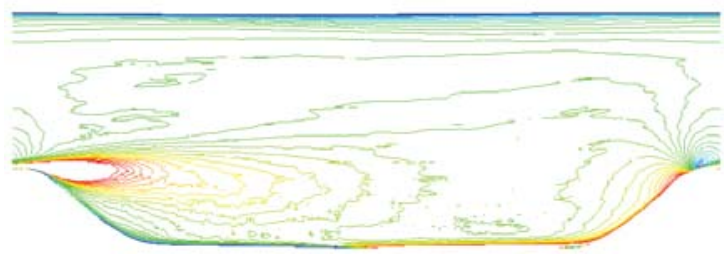

(c) $s_{12}$

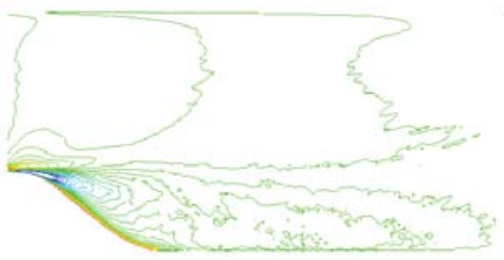

(e) $s_{22}$

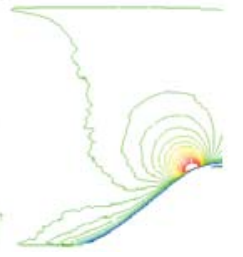$$
\text { (1) }
$$

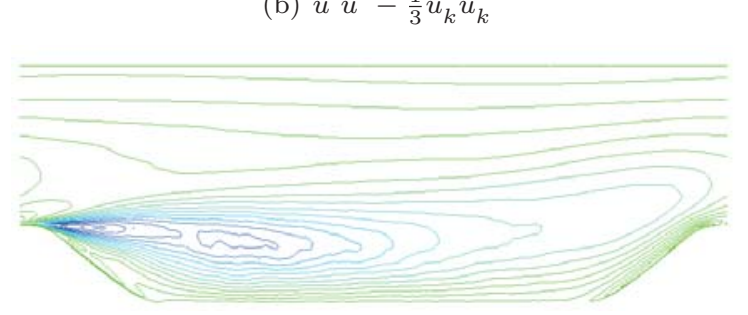

(d) $\overline{u^{\prime} v^{\prime}}$

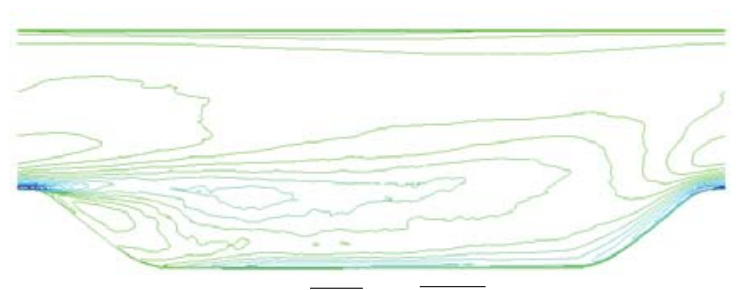

(f) $\overline{v^{\prime} v^{\prime}}-\frac{1}{3} \overline{u_{k}^{\prime} u_{k}^{\prime}}$

Figure 22. Reynolds stress and mean strain contours for the periodic hill at $R e=10,595$. (Contour lines correspond 30 uniform intervals between $[-2,2]$ for strain and $[-0.04,0.04]$ for Reynolds stresses.) 
were consistent with results previously presented in the literature.

Finally, we performed direct numerical simulation of turbulent flow in a channel with periodic hill constrictions. We validated our results by comparing with previously computed LES simulations. We have presented detailed statistics showing velocity distributions at various regions of the flow. Analysis of the resulting flow fields could provide insights that would facilitate improvements to RANS models. Future work will involve performing higher-fidelity simulations over larger times to provide statistically converged data. data.

\section{Acknowledgments}

Laslo Diosady was funded by the Revolutionary Computational Aerosciences sub-project within NASA's Fundamental Aeronautics program, through an appointment to the NASA Postdoctoral Program at the Ames Research Center, administered by Oak Ridge Associated Universities.

\section{References}

${ }^{1}$ Almeida, G., Durao, D., and Heitor, M., "Wake flows behind two-dimensional model hills," Exp Thermal Fluid Sci, Vol. 7, 1993, pp. 87-101.

${ }^{2}$ Mellen, C., Frohlich, J., and Rodi, W., "Large-eddy simulation of the flow over periodic hills," Proceedings of 16th IMACS world congress, edited by M. Deville and R. Owens, 2000.

${ }^{3}$ Fröhlich, J., Mellen, C. P., Rodi, W., Temmerman, L., and Leschziner, M. A., "Highly resolved large-eddy simulation of separated flow in a channel with streamwise periodic constrictions," J. Fluid Mech., Vol. 526, 2005, pp. 19-66.

${ }^{4}$ Peller and Manhart, "Turbulent channel flow with periodic hill constrictions," Arbeitsgemeinschaft Strömung mit Ablösung (STAB), edited by Mitteilungen, 2005, pp. 178-179.

${ }^{5}$ Breuer, M., Peller, N., Rapp, C., and Manhart, M., "Flow over periodic hills - Numerical and experimental study in a wide range of Reynolds numbers," Computers and Fluids, Vol. 38, 2009, pp. 433-457.

${ }^{6}$ Wang, Z., Fidkowski, K., Abgrall, R., Bassi, F., Caraeni, D., Cary, A., Deconinck, H., Hartmann, R., Hillewaert, K., Huynh, H., Kroll, N., May, G., Persson, P.-O., van Leer, B., and Visbal, M., "High-Order CFD Methods: Current Status and Perspective," International Journal for Numerical Methods in Fluids, Vol. 72, 2013, pp. 811-845.

${ }^{7}$ Orszag, S. and Patterson, G.S., J., "Numerical simulation of turbulence," Statistical Models and Turbulence, edited by M. Rosenblatt and C. Atta, Vol. 12 of Lecture Notes in Physics, Springer Berlin Heidelberg, 1972, pp. 127-147.

${ }^{8}$ Hughes, T. J. R., Franca, L., and Mallet, M., "A new finite element formulation for computational fluid dynamics: I Symmetric forms of the compressible Euler and Navier-Stokes equations and the second law of thermodynamics," Vol. 54, 1986, pp. 223-234.

${ }^{9}$ Ismail, F. and Roe, P. L., "Affordable, Entropy-consistent Euler flux functions II: entropy production at shocks," J. Comput. Phys., Vol. 228, No. 15, Aug. 2009, pp. 5410-5436.

${ }^{10}$ Bassi, F. and Rebay, S., "GMRES discontinuous Galerkin solution of the compressible Navier-Stokes equations," Discontinuous Galerkin Methods: Theory, Computation and Applications, edited by K. Cockburn and Shu, Springer, Berlin, 2000, pp. 197-208.

${ }^{11}$ Diosady, L. T. and Murman, S. M., "Design of a variational multiscale method for turbulent compressible flows," AIAA Paper 2013-2870, 2013

12 van Ress, W., Leonard, A., Pullin, D., and Koumoutsakos, P., "A comparison of vortex and pseudo-spectral methods for the simulation of periodic vortical flows at high Reynolds number," Journal of Computational Physics, Vol. 230, 2011, pp. 2794-2805.

${ }^{13}$ DeBonis, J. R., "Solutions of the Taylor-Green vortex problem using high-resolution explicit finite difference methods," AIAA Paper 2013-0382, 2013.

${ }^{14}$ Kim, J., Moin, P., and Moser, R., "Turbulence statistics in fully developed channel flow at low Reynolds number," Journal of Fluid Mechanics, Vol. 177, 1987, pp. 133-166.

${ }^{15}$ Eswaran, V. and Pope, S., "An examination of forcing in direct numerical simulations of turbulence," Computers and Fluids, Vol. 16, 1988, pp. 257-278. 